\title{
Thin-film metal oxides in organic semiconductor devices: their electronic structures, work functions and interfaces
}

\author{
Mark T Greiner and Zheng-Hong Lu
}

Thin-film metal oxides are among the key materials used in organic semiconductor devices. As there are no intrinsic charge carriers in a typical organic semiconductor, all charges in the device must be injected from electrode/organic interfaces, whose energetic structure consequentially dictates the performance of devices. The energy barrier at the interface depends critically on the work function of the electrode. For this reason, various types of thin-film metal oxides can be used as a buffer layer to modify the electrode work function. This paper provides a review on recent progress in metal oxide/organic interface energetics, oxide valence structure and work function, as well as the impact of defects and interfacial reactions on oxide work functions. This review provides a rational guide to process engineers in selecting the best suitable electrode/oxide structures for a targeted applications.

NPG Asia Materials (2013) 5, e55; doi:10.1038/am.2013.29; published online 19 July 2013

Keywords: charge-injection layers; metal oxides; organic electronics

\section{INTRODUCTION TO ORGANIC ELECTRONICS}

The past three decades have seen major improvements in organic semiconductor devices. Technologies such as organic light-emitting diodes (OLEDs), organic photovoltaics (OPVs) and organic fieldeffect transistors (OFETs) have been steadily evolving and have begun to penetrate the mainstream consumer electronics market.

OLED displays are arguably the most mature of the organic semiconductor technologies. Ultra-high-resolution OLED displayswith high brightness, extreme contrast ratios, rich color reproduction and extended operating lifetimes-are already available in the consumer electronics market. The next major developments in OLED technology will likely be in the sector of high-efficiency illumination products, where improvements are still needed in terms of colorrendering index, high efficiency at high brightness and operating lifetime.

OPVs have also seen great progress in recent years, with powerconversion efficiencies increasing from $2.5 \%$ in 2001 to $9.2 \%$ in 2012. ${ }^{1,2}$ Commercial products are beginning to emerge in the consumer electronics market, and as operational lifetimes and power-conversion efficiencies continue to improve, there is good reason to believe that OPVs will continue to increase their share of the market in coming years.

Major developments have also been seen with OFETs-a technology that enables computer processors and display devices to take the form of flexible plastic sheets, using inexpensive production techniques. ${ }^{3,4}$ There is even the possibility of light-emitting OFETs that allow display devices to be designed without the need for independent display drivers. ${ }^{5}$ With recent improvements in driving voltages, switching speeds, on-off ratios, power consumption and operating lifetimes, these devices will likely show up soon in niche, mobile communication markets. ${ }^{6-10}$

The rapid development of organic electronics over the past few decades is a result of a large-scale, concerted effort from the science and engineering research community. One of the major areas of development has been in the field of interfacial engineering.

Organic electronic devices are generally composed of multiple layers of various materials. During device operation, charge carriers must pass through these materials, and therefore electrons must cross several interfaces along their paths. The interfaces often possess an energetic barrier that hinders the passage of charge carriers. Such 'interfacial charge-injection barriers' give rise to high driving voltages and low power efficiencies in organic electronic devices, thereby reducing device lifetime and negating the possibility of lightweight high-efficiency portable electronic devices.

One of the most important types of interface in an organic device is the interface between an electrode material and an organic semiconductor. These interfaces must allow charge carriers (either electrons or holes) to easily pass across the material boundary with minimal resistance.

Low-resistance electrode/organic interfaces are not as easy to achieve as one might think. The need to solve this problem gave rise to the field of interfacial engineering in organic electronics. ${ }^{11,12}$ 
Interfacial engineering often involves tailoring the electron energy levels of the materials that are in contact with one another, in order to minimize or eliminate the charge-injection barrier.

The magnitude of a charge-injection barrier depends on the relative positions of donor and acceptor energy levels on either side of an interface. ${ }^{11,13-15}$ The alignment between donor and acceptor levels is often referred to as energy-level alignment (ELA). For an organic semiconductor, the donor level is the highest-occupied molecular orbital (HOMO) and acceptor level is the lowest unoccupied molecular orbital (LUMO). For conductive electrodes, the Fermi level $\left(E_{\mathrm{F}}\right)$ serves as both the donor and acceptor level.

The ELA criteria vary slightly for different types of organic devices, as illustrated in Figure 1. In OLEDs, there are two types of chargeinjection contacts; electron-injecting and hole-injecting contacts. Low-resistance electron-injecting contacts require that the donor level of the electrode (that is, the cathode) be closely aligned with the LUMO level of the organic semiconductor. Conversely, low-resistance hole-injecting electrodes (that is, anodes) require that the electrode's acceptor level (that is, Fermi level) is closely matched with the organic's HOMO level.

In $p$-OFETs, there are hole-injecting contacts (Figure 1c), where the Fermi level of the source electrode should align with the HOMO of the organic semiconductor to minimize the hole-injection barrier. In $n$-OFETs, there are electron-injecting contacts (Figure 1d), where the Fermi level of the source electrode should align with the LUMO of the organic semiconductor to minimize the electron-injection barrier.

With OPV's, the scenario is slightly different (Figure 1b). One often refers to hole-collecting and electron-collecting electrodes, rather than hole-injecting and electron-injecting electrodes. A hole-collecting electrode should have its donor level closely aligned with the organic's
HOMO level, and an electron-collecting electrode should have its acceptor level closely aligned with the organic's LUMO level to minimize energy losses.

Interface engineering, in the context of organic electronics, involves tailoring the ELA between the electrode and the organic material, often via some sort of surface treatment to the electrode material, or by incorporating a thin buffer layer between the electrode and the organic. Common surface treatments include halogenation, ${ }^{16-20}$ plasma reactions, ${ }^{21-27} \mathrm{UV}$-ozone ${ }^{28-30}$ and controlled air exposure. ${ }^{31-36}$ Buffer layers include vapor-deposited small molecules, ${ }^{37-39}$ spincast polymers, ${ }^{40-46}$ inorganic salts, ${ }^{12,47-52}$ covalently bound selfassembled monolayers ${ }^{53-58}$ and thin metal oxide layers. ${ }^{59-91}$ This review concentrates on the use of transition metal oxides as buffer layers in organic electronics.

\section{TRANSITION METAL-OXIDE BUFFER LAYERS}

Transition metal oxides are particularly versatile materials for use as buffer layers, as they can be used to achieve efficient charge injection for nearly any type of electrode. The first reported use of oxide buffer layers in OLEDs was by Tokito et al. ${ }^{61}$ in 1996. Now, transition metal oxides are heavily utilized in OLEDs, ${ }^{60}$ OPVs, ${ }^{92-97}$ and OFETs,,${ }^{98-104}$ and are components in many of the current record-breaking devices reported in the literature. ${ }^{2,105}$

Transition metal oxides can possess a wide range of work functions, spanning from extreme low of $3.5 \mathrm{eV}$ for defective $\mathrm{ZrO}_{2}$ to the extreme high of $7.0 \mathrm{eV}$ for stoichiometric $\mathrm{V}_{2} \mathrm{O}_{5} .{ }^{106}$ High-workfunction metal oxides are often used as hole-injecting buffer layers for anodes, ${ }^{60,61}$ including $\mathrm{MoO}_{3},{ }^{61-71} \mathrm{WO}_{3},{ }^{72,73,107} \mathrm{NiO},{ }^{74-81,108}$ $\mathrm{CuO},{ }^{82-83} \mathrm{RuO}_{2},{ }^{61} \mathrm{~V}_{2} \mathrm{O}_{5},{ }^{61,84-86} \mathrm{Fe}_{3} \mathrm{O}_{4}{ }^{87}$ and $\mathrm{Ag}_{2} \mathrm{O} .{ }^{88,109}$ The lowwork-function transition metal oxides-such as $\mathrm{TiO}_{2},{ }^{89} \mathrm{ZnO}^{90,104,110}$
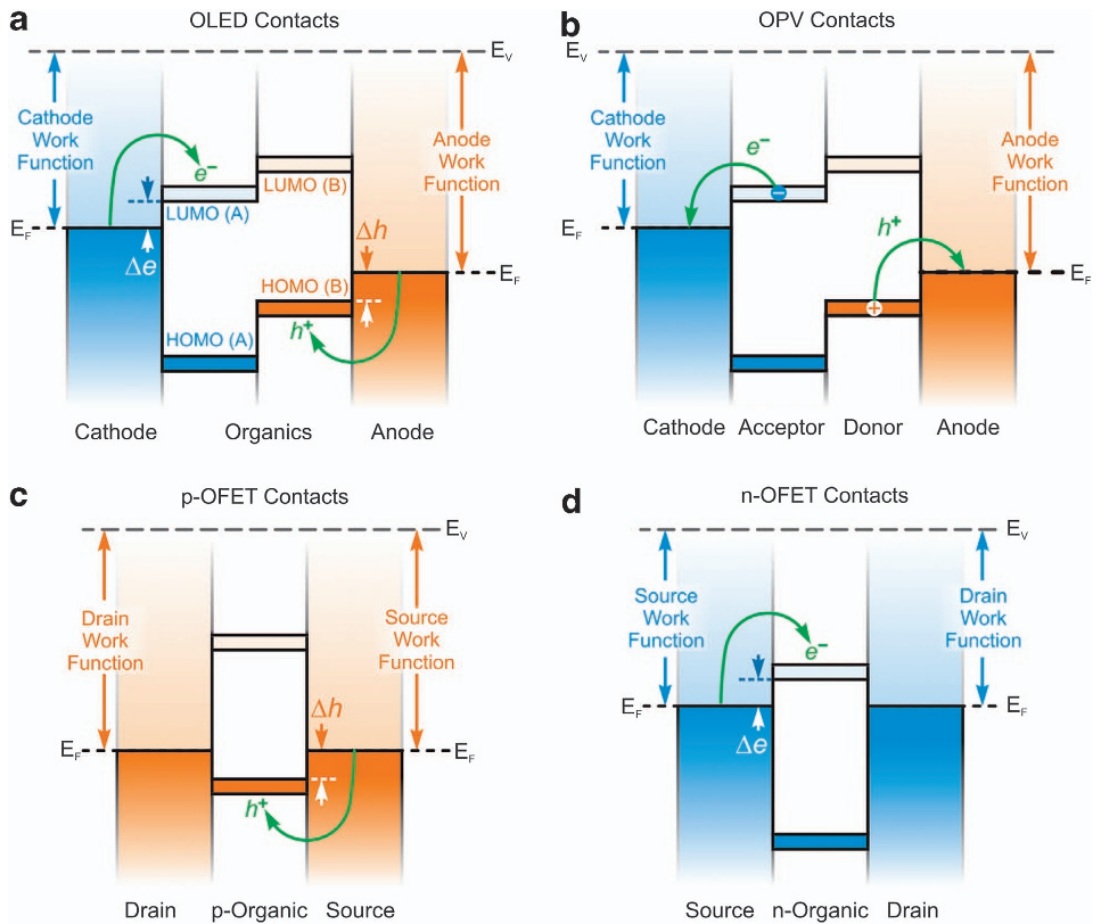

Figure 1 Schematic energy-level diagram of (a) a two-layered OLED, (b) a two-layered OPV, (c) an one-layer $p$-OFET and (d) an one-layer $n$-OFET. The green arrows indicate the movement of electrons $\left(e^{-}\right)$and holes $\left(h^{+}\right)$. The electron-injection barrier $(\Delta e)$ and hole-injection barrier $(\Delta h)$ are indicated. $E_{\mathrm{v}}$ represents the vacuum level. In an OLED, electrons and holes move from the Fermi levels $\left(E_{\mathrm{F}}\right)$ of the electrodes to the LUMO and HOMO levels, respectively. In an OPV, excited electrons and holes move from the LUMO of the acceptor and the HOMO of the donor, respectively, to the electrode Fermi levels. In OFETs, holes move from the source Fermi level into the organic HOMO level (in the case of $p$-OFETs) and from the source Fermi level into the organic LUMO level (in the case of $n$-OFETs). 
and $\mathrm{ZrO}_{2}{ }^{91,111}$ - are used as electron-injection buffer layers for cathodes. Some oxides can be evaporated at relatively mild temperatures-such as $\mathrm{MoO}_{3}, \mathrm{WO}_{3}$ and $\mathrm{V}_{2} \mathrm{O}_{5}$ - and are used for devices that are fabricated in vacuum. Other oxides can be solution deposited-such as $\mathrm{NiO},{ }^{80,112} \quad \mathrm{~V}_{2} \mathrm{O}_{5},{ }^{96,113} \quad \mathrm{TiO}_{2},{ }^{89,114} \quad \mathrm{WO}_{3},{ }^{95}$ $\mathrm{ZnO},{ }^{115,116} \mathrm{MoO}_{3}{ }^{117}$ and sub-stoichiometric $\mathrm{MoO}_{x}{ }^{118}$-making them convenient to use as buffer layers in solution-processed organic devices. ${ }^{117}$ There has also been some use of substoichiometric oxides, such as $\mathrm{WO}_{3-x},{ }^{119,120}$ and $\mathrm{MoO}_{3-x},{ }^{118,121-124}$ which tend to be metallic and can provide low-resistance buffer layers.

ELA AND THE IMPORTANCE OF ELECTRODE WORK FUNCTION It has been known since the early studies on organic interfaces that electrode work function is an important parameter for ELA. ${ }^{11,125-129}$ However, because of the complexity of interfaces, it has taken decades for the picture of interfacial ELA to become clear.

Electrode/organic interfaces appear to fall into three classifications: (1) chemically reactive/strongly interacting interfaces, (2) moderately interacting interfaces and (3) nonreactive/weakly interacting interfaces. One cannot draw a distinct dividing line that separates one class from another, but rather the classes likely span a continual range from strong chemical interactions to weak chemical interactions. Yet, there are some features that distinguish the three classes; in particular, their ELA behavior.

Chemically reactive or strongly interacting interfaces give rise to either breaking and reforming of chemical bonds in the organic or the formation of localized chemical bonds by strong hybridization between electrode surface electronic states and molecular orbitals (MOs). ${ }^{130-136}$ There are numerous examples from adsorption studies that demonstrate interfaces in which hybridization between adsorbate and substrate electronic states gives rise to distinct interface states. Work done between the 1970s and present day has provided many examples of such interfaces, involving small organic molecules on metals, ${ }^{131,132,134,136-150}$ noble gas atoms on metals, ${ }^{151-157}$ small inorganic molecules (such as $\mathrm{CO},{ }^{158-164} \mathrm{NO}^{165}$ and $\mathrm{H}_{2} \mathrm{O}^{166,167}$ ) on metals and oxides, ${ }^{160,161}$ and various organic semiconductors on metals and inorganic semiconductors. ${ }^{131,132,134,136,139,140,145-150}$ These interfaces usually involve reactive metals, or planar molecules that can come in very close contact with metal electrodes. The ELA behavior of these interfaces is complex, involving many considerations such as molecular adsorption geometry and the overlap integrals between molecule and substrate electron wave functions. No simple relationship is known that can generalize the ELA behavior at such interfaces.
Moderately interacting interfaces also involve hybridization between substrate and molecular wave functions, except the hybridization gives rise to interface states and broadening of MOs, rather than localized chemical bonds. ${ }^{127,128,168-172}$ These types of interfaces are common for nonreactive metal electrodes, and can result in a wide range of interaction strengths. This type of interface has been thoroughly examined both theoretically and experimentally. ELA behavior is governed by both electrode work function and interaction strength.

Nonreactive or weakly interacting interfaces have essentially no hybridization between the electrode bands and the organic orbitals. Consequently, there are no localized bonds and likely also no gap states at these interfaces. ELA behavior in these cases is mainly governed by electrode work function and organic donor/acceptor levels. ${ }^{173,174}$

Many metal-oxide/organic interfaces are weakly interacting interfaces. ${ }^{174}$ Weakly interacting interfaces give rise to perhaps the simplest ELA trend, which is illustrated in Figure 2b. The blue dashed line represents the electron-injection barrier $(\Delta e)$ and the orange line represents the hole-injection barrier, according to Figure $2 \mathrm{a}$. The horizontal axis in Figure $2 \mathrm{~b}$ is electrode work function. Figure $2 \mathrm{c}$ shows experimental measurements of the trend.

The trend shows that, within a certain range, the hole-injection and electron-injection barriers are linearly dependent on an electrode's work function. This linear region is referred to as the 'vacuum-level alignment' regime (although the vacuum levels of electrodes and organics do not always align perfectly here). In this region, increasing an electrode's work function decreases the hole-injection barrier and increases the electron-injection barrier. Conversely, decreasing an electrode's work function will increase the hole-injection barrier and decrease the electron-injection barrier.

The linear relationship between HOMO binding energy and electrode work function occurs because the surface potential of the electrode is proportional to the work function. In the absence of charge transfer, the position of an adsorbed molecule's frontier orbitals relative to the Fermi level depends on the electrode's surface potential.

If the electrode's work function exceeds a certain threshold valuea value that depends on the organic molecule - then the HOMO level becomes 'pinned' to the Fermi level. Note that 'pinning' means that the alignment between the HOMO and the Fermi level stays constant with further increases in electrode work function. Conversely, if an electrode's work function is lower than another threshold value, the Fermi level becomes 'pinned' to the LUMO level, and the alignment
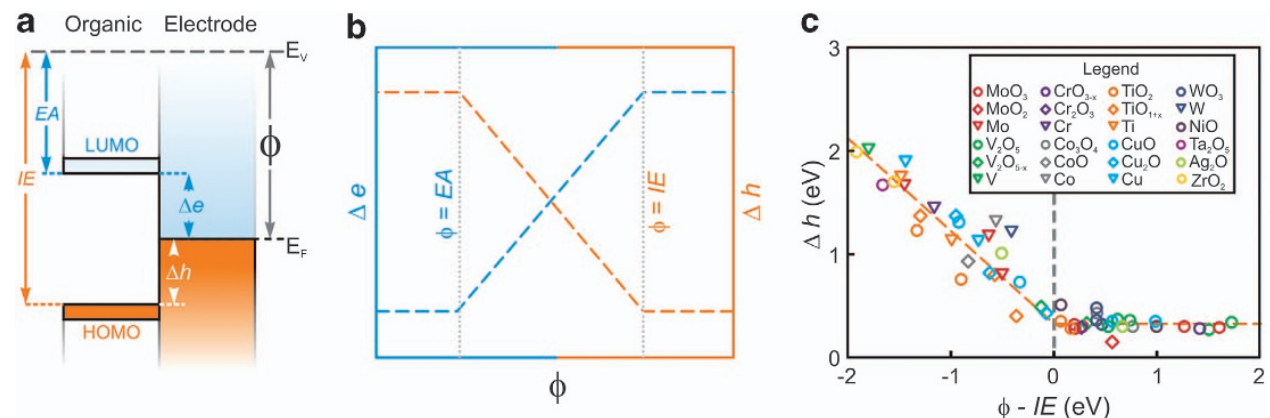

Figure 2 (a) Schematic energy-level diagram of an organic/electrode interface, where IE and EA are the organic's ionization energy and electron affinity, respectively. $\Delta h$ and $\Delta e$ are the hole- and electron-injection barriers, respectively. $E_{\mathrm{F}}$ is the Fermi level, $E_{\mathrm{v}}$ is the vacuum level and $\phi$ is the electrode's work function. (b) An illustration of the energy-alignment trend for weakly interacting systems, showing how hole-injection barrier and electron-injection barrier depend on work function, ionization energy and electron affinity. (c) Experimental photoemission measurements of energy-level alignment at the anode interface for several organic semiconductors on numerous metals and metal oxides. ${ }^{174}$ 
between the Fermi and LUMO level does not change with further decreases in electrode work function.

The onset of pinning is believed to occur as a result of molecular ionization at the electrode/organic interface and capacitive effects in the electrode. ${ }^{173-175}$ Because of coupling between the molecular ionization processes and the electrode's Fermi energy, ionized molecules become thermodynamically stable at the electrode surface once the Fermi level crosses the threshold to the 'pinning' regime. The electric potential-caused by the combined effects of ionized molecules at the interface and capacitive charge redistribution in the substrate-counteracts the surface potential that is related to work function. ${ }^{174,175}$

This ELA trend was first observed by Tengstedt et al. ${ }^{173}$ using polymer/polymer interfaces. The trend has now been confirmed by several other groups, using both photoemission spectroscopy and Kelvin probe methods. ${ }^{174,176-179}$ Greiner et al. ${ }^{174}$ demonstrated that the trend also holds for small-molecule organic semiconductors on a wide range of transition metal oxides.

As mentioned earlier, the threshold values that govern the onset of pinning depend on the characteristics of the organic molecule. Although Tengstedt et al's ${ }^{173}$ study showed that the threshold to pinning occurs when the electrode's work function is greater than the polymer's positive polaronic level and less than its negative polaronic level, Greiner et al.'s study found that the threshold to pinning occurs when the electrode's work function exceeds the organic molecule's ionization energy and electron affinity. The discrepancy may be because Tengstedt et al's study used solution-cast conducting polymers, whereas Greiner's study used vapor-deposited smallmolecule organic semiconductors.

Polaronic levels are related to ionization energy and electron affinity, but polaronic levels contain an additional energy term that results from the electron-density distortions that occur when highly polarizable molecules become charged. ${ }^{173}$ The positive polaronic level represents the sum of the polymer's ionization energy and the relaxation energy caused by molecular polarization. Likewise, the negative polaronic level represents the sum of the electron affinity and a relaxation energy.

Thus, it appears that for large and highly polarizable polymeric molecules, in which relaxation effects are large, one must consider the polaronic levels for the pinning thresholds. Although for small molecular semiconductors, in which polarization effects are considerably smaller, the relaxation term can be neglected and one needs to only consider a molecule's ionization energy/electron affinity to determine the pinning thresholds.

Experimentally, the pinning trend has been shown very extensively for the hole-injection side of the plot, as shown in Figure $2 \mathrm{c}$ by

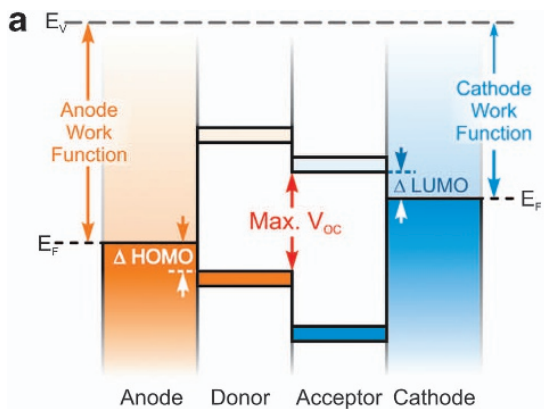

measuring the HOMO level of organics on high-work-function electrodes using photoemission spectroscopy. The low-work-function end of the plot has had less experimental confirmation, likely due to the scarcity of materials with low-enough work function to pin the LUMO levels of common organic semiconductors, while simultaneously being nonreactive. Furthermore, occupied levels are more straightforward to measure than unoccupied levels. Ultraviolet photoemission spectroscopy is commonly used to measure the HOMO level. To date, there have been no studies that use inverse photoemission to observe the pinning trend by measuring the LUMO level.

\section{IMPLICATIONS OF ELA BEHAVIOR}

The ELA behavior of weakly interacting interfaces has important implications for organic device design. For instance in OLEDs and OTFTs, when choosing an electrode material (or buffer layer) to give an Ohmic hole-injection contact between the anode and a given organic semiconductor, one must ensure that the electrode's work function is greater than the organic's ionization energy/positive polaronic level. Conversely, when choosing an appropriate cathode material to have an Ohmic electron-injection contact, one must ensure that the cathode's work function is lower than the organic's electron affinity/negative polaronic level.

In designing OPVs, the ELA trend has implications not only for contact resistance but also for maximizing open-circuit voltage $\left(V_{\mathrm{OC}}\right)$. The maximum $V_{\mathrm{OC}}$ that an organic solar cell can achieve depends on the offset between a donor molecule's HOMO and an acceptor molecule's LUMO. ${ }^{6}$ However, the maximum $V_{\mathrm{OC}}$ can only be achieved by appropriately tuning the ELA at the electrode/organic interfaces.

As shown in Figure 3, the maximum $V_{\mathrm{OC}}$ is achieved by tuning the electrode work functions, such that the donor's HOMO level and acceptor's LUMO level are pinned to their respective electrodes. If this is the case, the maximum $V_{\mathrm{OC}}$ will depend on the energy offset between the donor's HOMO level and the acceptor's LUMO level. However, if HOMO and LUMO levels are not pinned to their respective electrodes, then the $V_{\mathrm{OC}}$ will be less than ideal and will scale with the electrode work-function difference.

\section{GENERAL ELECTRONIC STRUCTURE OF METAL OXIDES}

Although transition metal oxides have been found to follow a general ELA behavior, their diverse set of electronic structures affects their conductivity properties. Transition metal oxides can be dielectric insulators, defective semiconductors ( $p$ - and $n$-type), Mott-Hubbard insulators and metallic conductors. These properties are established from a combination geometrical, quantum chemical and electrostatic

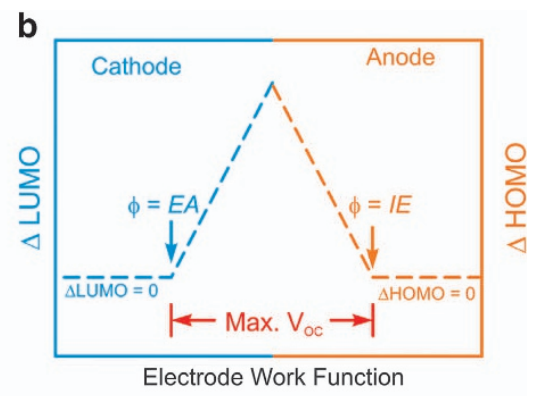

Figure 3 (a) Schematic energy-level diagram of an OPV, indicating the anode and cathode work functions, the HOMO and LUMO offsets ( $\triangle \mathrm{HOMO}$ and $\triangle \mathrm{LUMO}$, respectively) and the maximum open-circuit voltage $\left(V_{O C}\right)$. (b) Illustration of how the maximum open-circuit voltage depends on energy-level alignment. The blue dashed line represents the LUMO offset ( $\triangle$ LUMO), and the orange line represents the HOMO offset $(\triangle H O M O)$. 
characteristics. For example, crystal symmetry, metal-oxygen bond length, valence electron population, degree of ionic or covalent bond character and electron correlation effects, all influence a metal oxide's electronic properties. Although a quantitatively accurate description of an oxide's electronic structure often requires most of these factors to be considered, many of the qualitative features can be understood from rather simple models - such as crystal field theory or ligand field theory-and by simply considering $d$-band occupancy. ${ }^{180}$

In crystal field theory, one treats oxygen atoms as negative point charges. Conceptually, these point charges are positioned in a particular geometry around a metal atom, as illustrated in Figure 4 for the octahedral case. One then considers how the electric fieldwhich is formed by the negative point charges-affects the energy of the metal atom's $d$ electrons. Although a metal atom's $d$ orbitals are degenerate when they are surrounded by a spherically symmetric electric field (or zero electric field), they lose their degeneracy when surrounded by an electric field of lower symmetry than a sphere.

In crystal field theory (that is, in the ionic approximation), oxides are treated as though the metal atom has transferred all of its valence electrons to the oxygen atom. Thus, the oxygen atom is treated as an $\mathrm{O}^{2-}$ ion and the metal as an $\mathrm{M}^{x+}$ ion, where $x$ is the formal

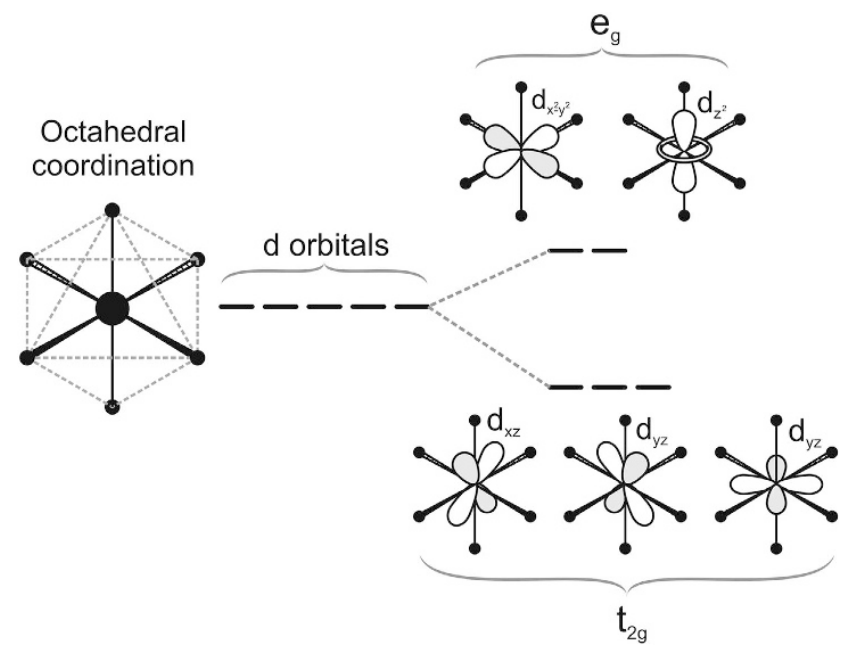

Figure 4 (left) An illustration of a metal ion that is surrounded by point charges in an octahedral geometry. (right) An illustration of how the energies of metal $d$ orbitals are split when octahedrally coordinated by negative point charges. oxidation state of the transition metal. This model represents the hypothetical scenario where there is zero hybridization between $\mathrm{O} 2 p$ and metal $d$ orbitals.

In most metal oxides, the oxygen anions are arranged in an octahedral or tetrahedral geometry around the metal cation. In an electric field of octahedral symmetry, two of the five $d$ orbitals are destabilized (pushed up in energy) and the other three $d$ orbitals are stabilized (pulled down in energy), as illustrated in Figure 4. This splitting of $d$-orbital energies creates an energy gap. The two orbitals that are destabilized are assigned the point-symmetry label $e_{\mathrm{g}}$, and the three stabilized orbitals are assigned the point-symmetry label $t_{2 \mathrm{~g}}$.

In contrast to the ionic approach of crystal field theory, ligand field theory is a MO approach, in which oxide energy levels are calculated from the linear combinations of atomic orbitals-mainly the metal $d$ and oxygen $2 p$ orbitals. For octahedrally and tetrahedrally coordinated metals, the computed MOs from ligand field theory yield similar structures as crystal field theory-that is, the $d$ orbitals are split into MO's of $e_{\mathrm{g}}$ and $t_{2 \mathrm{~g}}$ symmetry. An example of the electronic structure of $\mathrm{TiO}_{2}$, from $\mathrm{MO}$ overlap calculations, is shown in Figure 5.

The ligand field picture is often an adequate description of oxides-except when electron correlation effects are strong, in which case coulomb repulsion between electrons must be considered.

In extended solids, the energy levels from ligand field theory are broadened into bands in the same way that atomic orbitals of metals and semiconductors are broadened into bands in solids. Both $\mathrm{O} 2 p$ states and metal $d$ states contribute to the total density of states (DOS). Overlap integrals between oxygen $2 p$ states and metal $d$ states are usually nonzero, thus bands generally consist of a hybridized mixture of oxygen and metal states.

The total DOS can be divided into projected DOS plots, which show what portion of the total DOS is contributed from O $2 p$ states and metal $d$ states, as shown for anatase $\mathrm{TiO}_{2}$ in Figure 5b. Often a certain band can be said to be majority $\mathrm{O} 2 p$ character, or majority metal $d$ character, giving rise to the labeling of the $\mathrm{O} 2 p$ band or metal $d$ band. In the example of anatase $\mathrm{TiO}_{2}$, one can see that the occupied valence band is majority oxygen $2 p$ character, and the unoccupied conduction band is majority $\mathrm{Ti} 3 d$ character.

Although the finer details of transition metal-oxide electronic structures require computation, some of the most important qualitative features can be understood from the above-described models and considerations of the $d$-band occupancy. ${ }^{34}$ Figure 6 shows some transition metal-oxide valence spectra, which have been grouped into classes according to their $d$ occupancy. The top row of the Figure 6 presents each class's schematic energy-level diagram, and the bottom
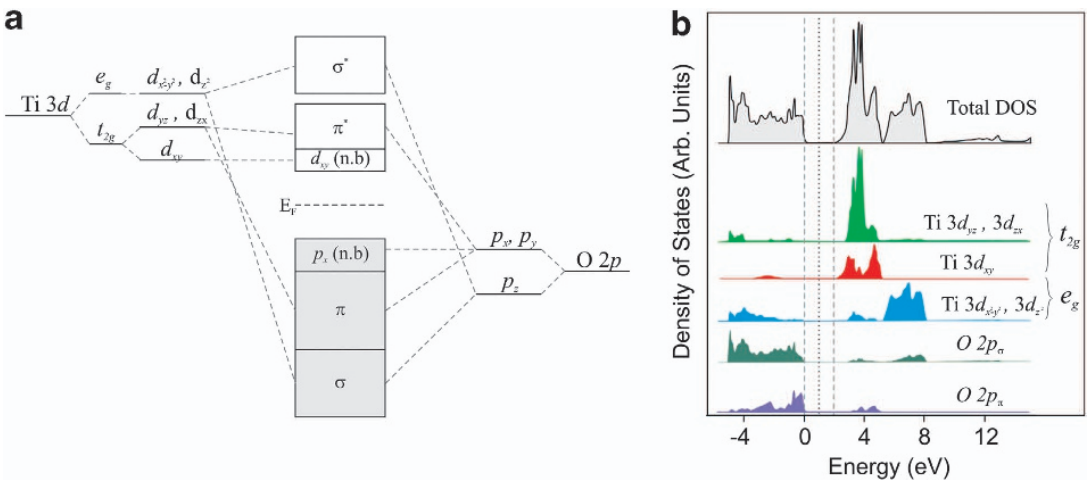

Figure 5 (a) Molecular orbital energy-level diagram of anatase $\mathrm{TiO}_{2}$ (adapted from Asahi et al. ${ }^{181}$ ). (b) Calculated total density of states (top) and projected density of states for $\mathrm{Ti} 3 d$ and $02 p$ orbitals (below), as labeled (adapted from Jiang et al. ${ }^{182}$ ). 

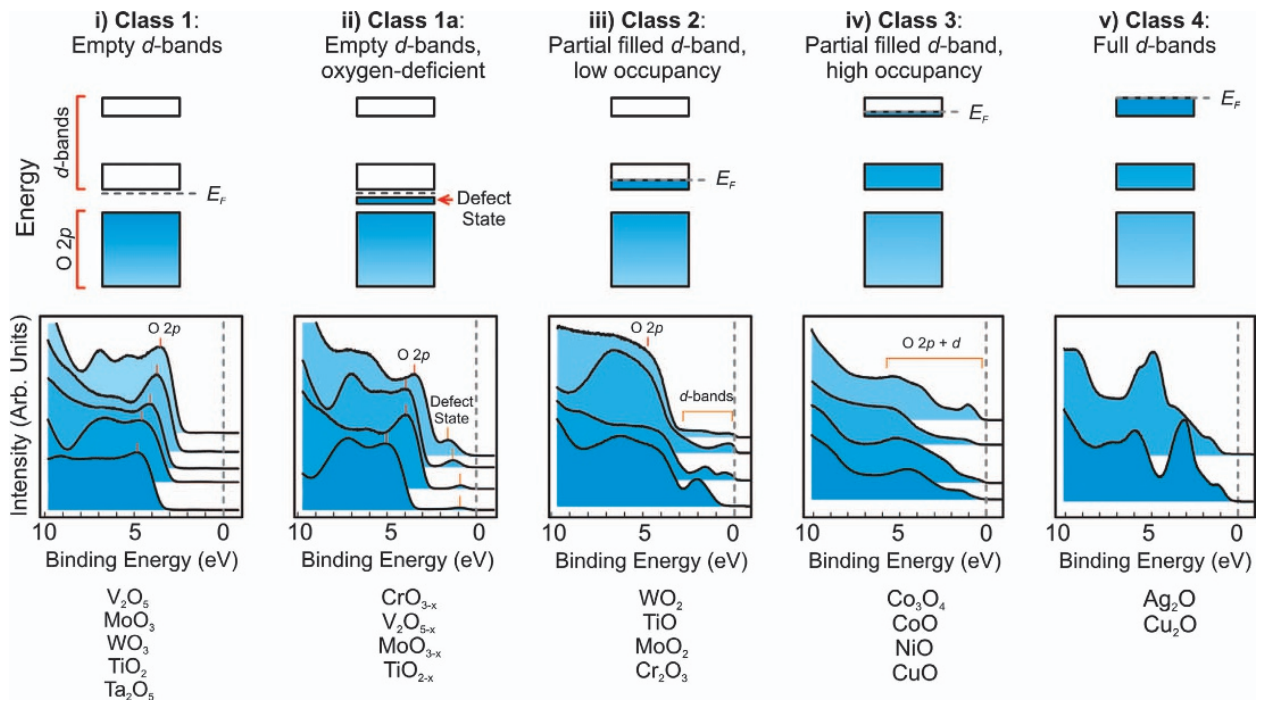

Figure 6 (top) Schematic energy-band diagrams and (bottom) valence-band photoemission spectra of various transition metal-oxide classes. The classes differ in terms of their $d$-band occupancy, ranging from completely empty (far left) to completely filled (far right). ${ }^{174}$

row presents their valence photoemission spectra. For simplicity, the different cation coordination geometries are not distinguished, but it is sufficient to say that, whatever the anion geometry, there is an energy gap in the $d$ band.

The class 1 oxides have totally empty $d$ bands ( $d^{0}$ oxides). When completely stoichiometric, these oxides are insulators. However, they have a natural tendency to form oxygen vacancy defects, and consequently they are $n$-type materials. ${ }^{34,40}$ The bottom of their conduction bands are composed primarily of empty metal $d$ states, and valence-band maxima are composed primarily of $\mathrm{O} 2 p$ states, as shown in Figure 6 (i). Examples of these oxides are: $\mathrm{MoO}_{3}, \mathrm{TiO}_{2}$, $\mathrm{HfO}_{2}, \mathrm{WO}_{3}, \mathrm{ZrO}_{2}, \mathrm{Ta}_{2} \mathrm{O}_{5}, \mathrm{~V}_{2} \mathrm{O}_{5}$ and $\mathrm{CrO}_{3}$.

A subset of the class 1 oxides is the oxygen-deficient $d^{0}$ oxides. With high a degree of oxygen deficiency, a significantly high density of occupied states form within the band gap. These defect states arise from the filling of empty metal $d$ states, and can be seen in the valence spectra of Figure 6 (ii). As a result of the small energy gap between the defect states and the conduction band minima, these oxides tend to be $n$-type semiconductors.

The class 2 oxides are oxides whose $d$ bands are partially occupied, but with a low number of electrons (for example, $d^{1}, d^{2}$ and $d^{3}$ ). These oxides tend to form when $d^{0}$ oxides are chemically reduced. They are often metallic, as evident by the finite DOS at the Fermi level in the valence spectra level in Figure 6 (iii).

The oxides whose $d$ bands are partially occupied with a high number of electrons (for example, $d^{7}, d^{8}$ and $d^{9}$ ) constitute class 3 oxides. These oxides represent a very important class of materials, called Mott-Hubbard or charge-transfer insulators. Their electronic properties are dominated by electron-electron correlations. The valence photoemission spectra of these oxides are not as simple to interpret as the $d^{0}$ oxides, because there is often a significant amount of hybridization between metal $d$ and oxygen $p$ states, thus one cannot easily divide the valence DOS into metal $d$ regions and oxygen $p$ regions. Furthermore, the strong electron correlation gives rise to several final-state photoemission features. Nonetheless, the valence photoemission spectra of the class 3 oxides share similar qualitative features, as seen in Figure 6 (iv).

Because of the strong electron correlation, the electronic properties of these oxides are not correctly predicted using the simple independent-electron model that is often used to describe band structures of solids. According to the independent-electron description, class 3 oxides should have a partially filled $d$ band and should therefore be metallic conductors. Contrary to this prediction, these oxides are electrical insulators. The electrical conductivity of these oxides can be explained by considering on-site Coulomb repulsion and electron exchange interactions, in the models developed by Mott ${ }^{183}$ and Hubbard. ${ }^{184-186}$

In the Mott-Hubbard model, $d$ electrons are localized on metal cations because there exists a strong repulsion energy between electrons in narrow $d$ bands. ${ }^{187}$ In this model, conduction is envisioned as $d$ electrons hopping from one metal site to another (that is, $d_{i}^{n} d_{j}^{n} \rightarrow d_{i}^{n-1}$ $d_{j}^{n+1}$, where the $i$ and $j$ indices indicate different metal sites). Coulomb repulsion and exchange interactions between electrons introduce an energetic penalty to placing two $d$ electrons on the same site.

A material is metallic if there exists vacant states at an energy infinitesimally higher than the highest-occupied state. The Coulomb repulsion energy increases the energy of the lowest unoccupied state, such that it no longer is infinitesimally higher in energy than the highest-occupied state. In the band picture, this scenario can be viewed as the formation of an energy gap in the $d$ band, as illustrated in Figure 7. The splitting of the $d$ band gives rise to the so-called lower Hubbard band and the upper Hubbard band. The bands are separated by the Hubbard parameter (U).

Strong electron correlation can also give rise to so-called 'chargetransfer' insulators. ${ }^{188}$ In these materials, the lowest-energy excitation is not a $d-d$ excitation, but rather a transition from a ligand site to a metal site (that is, $d_{i}^{n} L^{m} \rightarrow d_{i}^{n+1} L^{m-1}$, where $L$ indicates a ligand, or in the case of oxides, oxygen). The energy required to excite an electron from the highest-occupied oxygen state to the lowest unoccupied metal $d$ state is represented by the charge-transfer term, $\Delta$. If the Hubbard term (U) is much greater than the charge-transfer term $(\Delta)$, as illustrated in Figure $7 \mathrm{~b}$, then the material is described as a charge-transfer insulator, rather than a Mott-Hubbard. The oxides $\mathrm{NiO}, \mathrm{CuO}$ and $\mathrm{CoO}$ are believed to be charge-transfer insulators. Although electron correlation has a strong influence on the bulk electrical conductivity properties of these metal oxides, it is not yet clear to what degree electron correlation affects the charge-injection characteristics at metal-oxide/organic interfaces. 
a

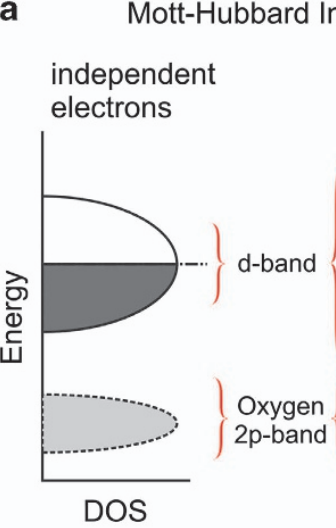

correlated electrons

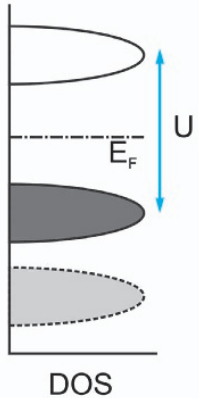

b

Charge-Transfer Insulator

independent electrons

correlated

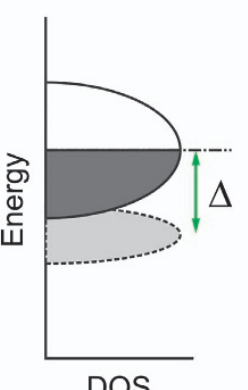

Figure 7 (a) Schematic energy-band diagrams of a Mott-Hubbard insulator, where the left side shows the 'independent-electron' model and the right side shows 'correlated-electron' model. (b) Schematic energy-band diagrams of a charge-transfer insulator. The occupied portion of the $d$ band is shaded in dark gray, and the occupied $02 p$ band is shaded in light gray and outlined with a dashed line. The Fermi level is indicated by a dotted-dashed line. The Hubbard term $(U)$ and charge-transfer term $(\Delta)$ are indicated in the figure. ${ }^{187}$

The final class of oxides discussed here (class 4 ) are the oxides with completely filled $d$ bands ( $d^{10}$ oxides). They tend to be semiconductors owing to the gap between the $d$ band and the next-highest energy band (usually derived from metal $s$ orbitals). These oxides tend to behave as intrinsic p-type semiconductors, where the Fermi level resides closer to the valence band than the conduction band; however, the hole mobility for these materials is still rather low. The reason for the $p$-type nature of these oxides is commonly accepted to be due to the presence of cation vacancy defects. ${ }^{189,190}$

\section{FERMI-LEVEL POSITION AND IMPORTANCE OF BAND STRUCTURE}

Hypothetically, the Fermi level of a perfectly stoichiometric semiconducting oxide would sit at midway between the valence-band maximum and the conduction band minimum. Realistically, a perfectly stoichiometric oxide (that is, an oxide without any defects) cannot exist, as entropy requires that there will always be some finite concentration of defects.

The common defects in oxides are oxygen vacancies $\left(v_{\mathrm{O}}\right)$ and metal vacancies $\left(v_{\mathrm{M}}\right)$, although other defects are also possible, such as metal or oxygen interstitials, an oxygen ion on a metal site and a metal ion on an oxygen site. In general, all of these defects give rise to either over-coordinated or under-coordinated metal centers, and result in new occupied or unoccupied states near the conduction band or valence-band edges. These defect states make an oxide behave as either a $p$ - or $n$-type semiconductor.

Using the simplified ionic picture, one can say that coordination defects result in metal oxidation-state defects (that is, metal centers where the metal's oxidation state differs from the value it would have in the stoichiometric oxide). An oxygen vacancy would give rise to metal cations with an extra electron. The extra electrons occupy states close to the conduction band minimum, and thus can act as $n$-type dopants. Conversely, a cation vacancy would result in unoccupied states close to the valence-band maximum, and can act as p-type dopants. In general, oxygen deficiency (or excess metal cations) makes an oxide behave as $n$-type, whereas metal deficiency (or excess oxygen) makes an oxide behave as p-type. Note that the actual position of a given occupied or unoccupied defect state, relative to the conduction and valence bands, differs from material to material, and in some oxides these states can act as traps.
Some metal oxides are described as 'intrinsically' $p$-type or 'intrinsically' $n$-type. This is simply because the formation energies of each type of defect are not equal. ${ }^{191}$ For most oxides, under normal conditions (that is, standard atmosphere, temperature and pressure), a given type of defect will tend to dominate. For instance, the most stable defect in $\mathrm{MoO}_{3}$ is the oxygen vacancy, and thus $\mathrm{MoO}_{3}$ will intrinsically behave as an $n$-type semiconductor. Conversely, the most stable defect in $\mathrm{Cu}_{2} \mathrm{O}$ is the $\mathrm{Cu}$ vacancy, and so intrinsically $\mathrm{Cu}_{2} \mathrm{O}$ behaves as a $p$-type semiconductor. ${ }^{192}$ In some oxides, the formation energies of various defects are similar in magnitude, so the oxide can change its behavior from $p$-type to $n$-type depending on the surrounding oxygen chemical potential. ${ }^{193-195}$ This behavior is rare, but is known to occur in the case of $\mathrm{UO}_{2} \cdot{ }^{193-195}$

An oxide's electronic structure is important to its use as a buffer layer. An oxide's charge-injection properties in a device will depend on whether the oxide is $p$ - or $n$-type, wide or narrow band gap, or a metallic conductor. Schematic energy-level diagrams of various transition metal oxides and several common organic semiconductors are shown in Figure 8.

As many of the oxides-due to intrinsic defects-are $p$ - or $n$-type semiconductors, it is possible for certain oxides to be used as selective charge-injection layers. A selective charge-injection layer decreases the injection barrier for only one type of carrier-a property that is particularly important for OPVs, in which nonselective contacts result in high leakage currents. ${ }^{196}$

There are numerous reports of transition metal oxide buffer layers in OPVs, where the oxide provides charge selectivity..$^{80,196-203}$ It is believed that the charge selectivity is a result of the $p$ - or $n$-type nature of a particular buffer layer. For instance, consider the $p$-type oxide anode buffer layer, illustrated in Figure 9b. The Fermi level is closer to the oxide's valence band than its conduction band. For a high-workfunction oxide, as would be the case for anode buffer layers, the Fermi level is also pinned to the HOMO level of the organic layer. Holes can easily hop through the buffer layer via the buffer layer's valence band. However, if the oxide's band gap is larger than the organic's HOMOLUMO gap, then there will be a barrier to overcome for any electrons trying to hop from the LUMO to the electrode.

The reverse is true for $n$-type oxides and cathode buffer layers. An $n$-type oxide has its conduction band close to the Fermi level. Furthermore, a low-work-function cathode has its Fermi level pinned to the LUMO level. Excited electrons in the LUMO will be able to 

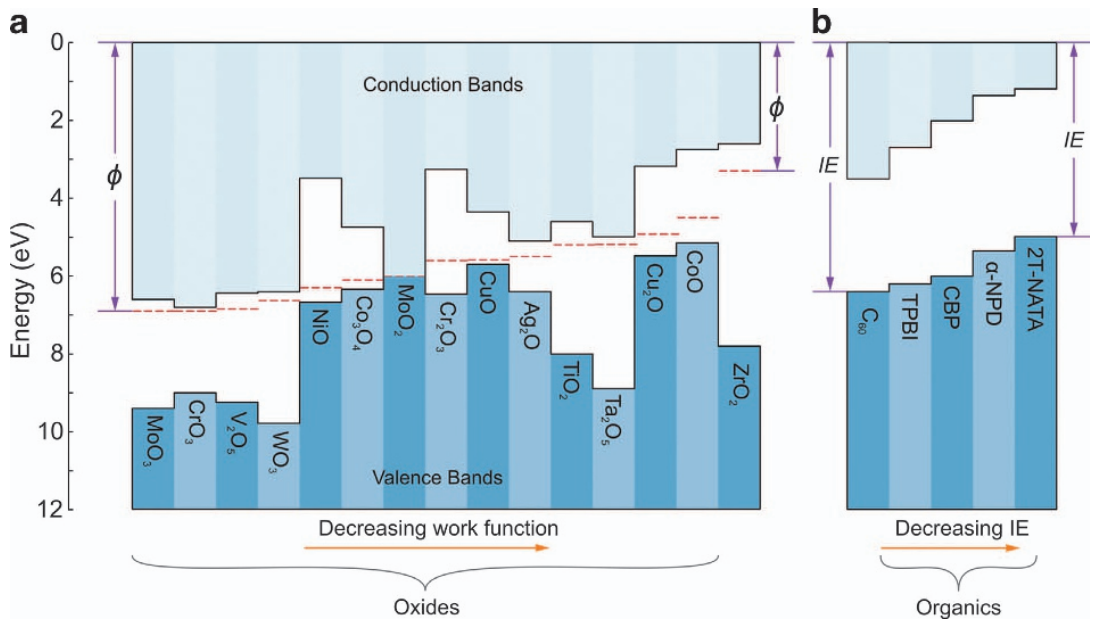

Figure 8 Schematic energy-level diagrams of (a) several transition metal oxides and (b) several organic semiconductors. The lower shaded regions represent the valence bands and the upper shaded regions represent the conduction bands. The dashed red lines indicate the position of the Fermi level for each oxide. Oxides with their Fermi levels close to the valence band are $p$-type semiconductors, and oxides with their Fermi levels close to the conduction band are $n$-type semiconductors. ${ }^{174}$

a

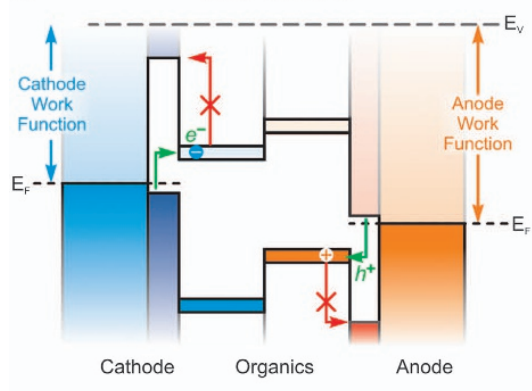

C

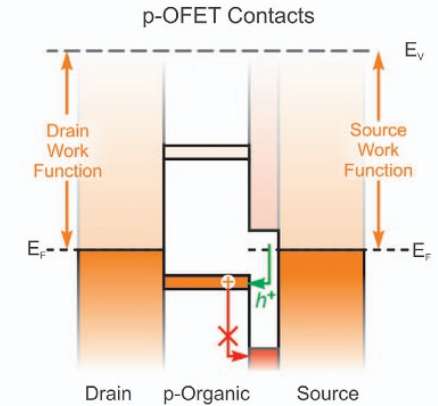

b

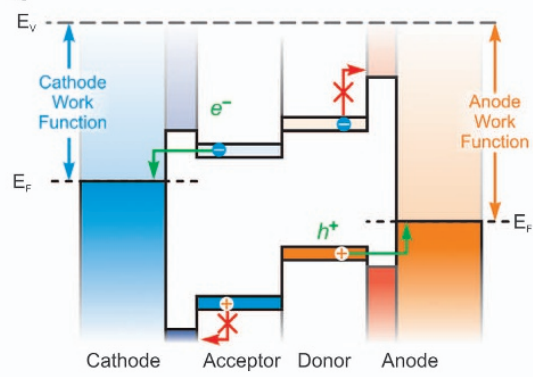

d

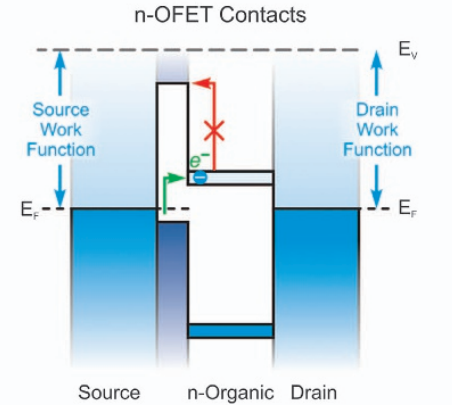

Figure 9 Schematic energy-level diagrams of charge-selective buffer layers at (a) OLED contacts, (b) OPV contacts, (c) p-OFET contacts and (d) n-OFET contacts. See text for further discussion.

move to the electrode via the buffer layer's conduction band. However, if the buffer's band gap is larger than the organic's HOMO-LUMO gap, then holes will have a barrier to overcome to move from the HOMO level to the electrode.

Consequently, the optimal buffer layer for the cathode of an OPV would be a low-work-function, $n$-type semiconductor with a large band gap, and the optimal buffer layer for an anode in an OPV would be a high-work-function, $p$-type semiconductor with a large band gap.

Similar rectifying behavior is believed to occur in OLEDs, as illustrated in Figure 9a. If the cathode has a wide band-gap p-type oxide as the buffer layer, then electrons can hop from the cathode to the organic LUMO level by overcoming the electron-injection barrier. However, the movement of electrons in the reverse direction requires electrons to overcome a much larger barrier (from the organic LUMO to the oxideconduction band).

Conversely, on the anode side, if a wide band-gap $n$-type oxide is used for the buffer layer, then there will be a small hole-injection barrier for holes moving from the anode to the organic HOMO level, and a large barrier for holes moving from the organic HOMO level to the oxide valence band. Similar behavior is expected for contacts in OFETs as illustrated in Figures $9 \mathrm{c}$ and $\mathrm{d}$. 


\section{DEPENDENCE OF OXIDE WORK FUNCTION ON OXIDATION STATES AND DEFECTS}

Given the role of work function in governing ELA, tuning work function is an important capability. However, a material's work function can be difficult to control. Work function values for a given material can span over $1 \mathrm{eV}$. For instance, reported work function values for $\mathrm{MoO}_{3}$ range from 5.3 to $6.9 \mathrm{eV} .32,64,92$

The large variation in reported work-function values is because work function is very sensitive to a number of factors. Work function represents the sum of electron chemical potential and surface dipole. Factors such as surface roughness, ${ }^{204,205}$ exposed crystal face, ${ }^{206}$ radiation exposure ${ }^{207,208}$ and adsorbate coverage ${ }^{209}$ affect the affected by the chemical identity of a material, the presence of impurities and the stoichiometry of a material. ${ }^{106}$

Although there are many factors that can alter work function, it is possible to gain control over many of these factors to tune work function. Under certain preparation conditions, one can obtain reproducible work-function values for a given material. For instance, sublimation-deposited $\mathrm{MoO}_{3}$ is routinely reported to have a work function of $c a .6 .8-6.9 \mathrm{eV}$.

A compilation of work-function values for various metals and metal oxides (prepared by in-situ metal oxidation) is provided in Table 1 . The work-function values in Table 1 represent the average of the most thermodynamically stable crystal faces of each oxide, as the

Table 1 Work-function values for sputter-cleaned metals and in-situ oxidized metal oxides, measured by photoemission spectroscopy

\begin{tabular}{lclclc}
\hline Material & $\phi(\mathrm{eV})$ & Material & $\phi(\mathrm{eV})$ & Material & $\phi(\mathrm{eV})$ \\
\hline $\mathrm{TiO}_{2}$ & $5.4 \pm 0.2$ & $\mathrm{TiO}$ & $4.7 \pm 0.2$ & $\mathrm{Ti}$ & $3.7 \pm 0.2$ \\
$\mathrm{MoO}_{3}$ & $6.82 \pm 0.05$ & $\mathrm{MoO}_{2}$ & $5.9 \pm 0.2$ & $\mathrm{Mo}$ & $4.4 \pm 0.2$ \\
$\mathrm{CuO}$ & $5.9 \pm 0.1$ & $\mathrm{Cu}_{2} \mathrm{O}$ & $4.9 \pm 0.1$ & $\mathrm{Cu}$ & $4.63 \pm 0.03$ \\
$\mathrm{NiO}$ & $6.3 \pm 0.2$ & - & - & $\mathrm{Ni}$ & $4.99 \pm 0.07$ \\
$\mathrm{WO}_{3}$ & $6.8 \pm 0.4$ & $\mathrm{~W}_{18} \mathrm{O}_{49}$ & $6.4 \pm 0.1$ & $\mathrm{~W}$ & $4.8 \pm 0.3$ \\
$\mathrm{~V}_{2} \mathrm{O}_{5}$ & $6.8 \pm 0.1$ & $\mathrm{~V}_{2} \mathrm{O}_{3}$ & 4.9 & $\mathrm{~V}$ & $4.0 \pm 0.2$ \\
$\mathrm{CrO}_{3}-x$ & $6.75 \pm 0.2$ & $\mathrm{Cr}_{2} \mathrm{O}_{3}$ & $5.0 \pm 0.5$ & $\mathrm{Cr}$ & $4.0 \pm 0.3$ \\
$\mathrm{Ta}_{2} \mathrm{O}_{5}$ & $5.2 \pm 0.2$ & - & - & $\mathrm{Ta}$ & $4.1 \pm 0.4$ \\
$\mathrm{Co}_{3} \mathrm{O}_{4}$ & $6.3 \pm 0.3$ & $\mathrm{CoO}_{0}$ & $4.6 \pm 0.2$ & $\mathrm{Co}$ & $4.8 \pm 0.2$ \\
\hline $\mathrm{ErOr}$ & & & & &
\end{tabular}

Error ranges represent the $95 \%$ confidence interval. ${ }^{106}$

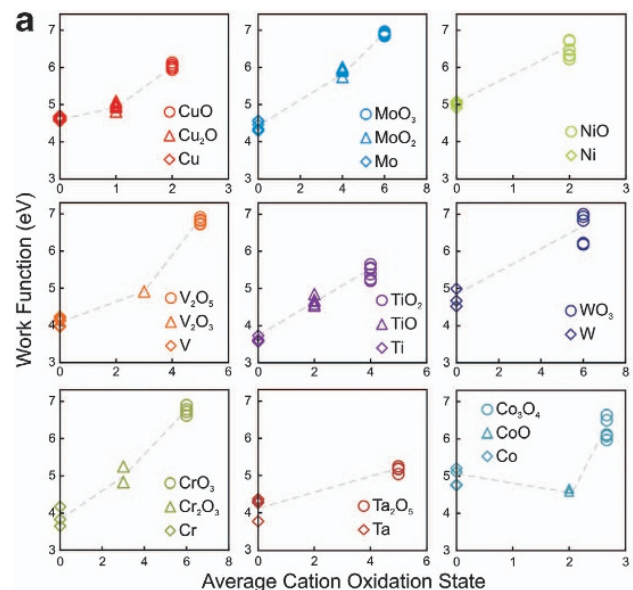
surface dipole component, whereas electron chemical potential is

oxides represented here were polycrystalline and prepared by in-situ metal oxidation. The oxides were not exposed to air, so adsorbates likely have a minimal effect on the work-function values shown here. Furthermore, as the oxides were prepared via in-situ oxidation (and vacuum annealing in the case of reduced oxides), they should all consist of stable oxygen-terminated surfaces. Consequently, the magnitude of the surface dipole in the work-function values listed in Table 1 can be considered similar in magnitude. Thus, the values allow an approximate comparison of relative electron chemical potential values.

A plot of metal-oxide (and metal) work functions versus average cation oxidation state shows that reducing the cation oxidation state of an oxide tends to decrease its work function, as shown in Figure 10a. This is likely a consequence of a decrease in electron chemical potential as the oxide becomes reduced (that is, as oxygen is removed). The reason for the decrease in electron chemical potential is that a cation's electronegativity decreases as its oxidation state decreases. $^{210}$

Electronegativity is closely related with the concept of electron chemical potential (or Fermi level). In Mulliken's definition, electronegativity represents the midway point between the ionization energy and electron affinity - that is, midgap, as shown in Figure 11a. ${ }^{211}$ Likewise, the Fermi level in undoped semiconductors is midgap, as shown in Figure $11 \mathrm{~b} .^{212}$ Thus, there is a direct relationship between electronegativity and Fermi level. ${ }^{213}$

Figure $10 \mathrm{~b}$ shows a plot of the midgap position for various oxides - as calculated using electronegativity — versus the experimental midgap position — as determined by UPS (ultraviolet photoemission spectroscopy) measurements and literature band gaps.

The midgap positions were calculated using the concept of group electronegativity. In a compound, the overall electronegativity (that is, the group electronegativity) can be calculated using the geometric mean of the electronegativity values of the individual components in the compound: ${ }^{213}$

$$
\chi_{\mathrm{AB}}=\left(\chi_{\mathrm{A}}^{m} \chi_{\mathrm{B}}^{n}\right)^{1 /(m+n)}
$$

Where $\chi_{\mathrm{AB}}$ is the group electronegativity of compound $\mathrm{A}_{m} \mathrm{~B}_{n}$, where $m$ and $n$ represent stoichiometric coefficients. $\chi_{\mathrm{A}}$ and $\chi_{\mathrm{B}}$ are the electronegativities of components A and $\mathrm{B}$, respectively. The concept of group electronegativity is illustrated in an energy-level diagram in Figure 11c.

b

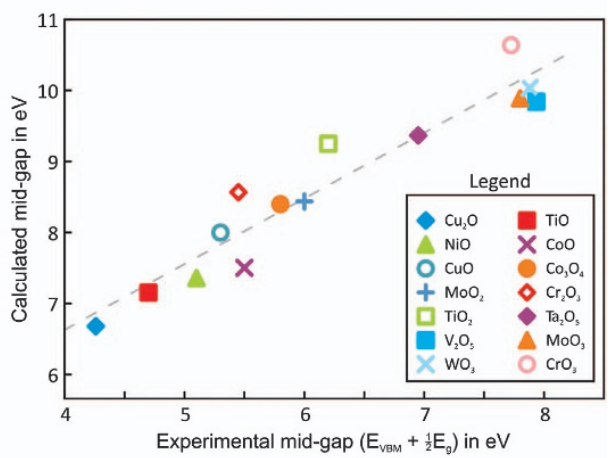

Figure 10 (a) Plots of work function versus nominal average oxidation state of metal atoms in several transition metals and transition metal oxides. (b) Calculated midgap positions (using ionic electronegativities) versus the experimental midgap position (determined from UPS measurements and literature band gaps) for several transition metal oxides. The dashed line shows the linear regression. ${ }^{106}$ 
a

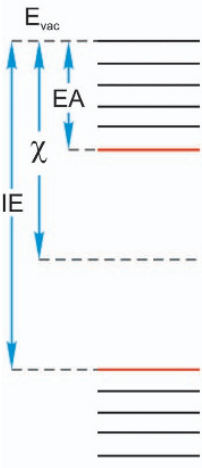

b

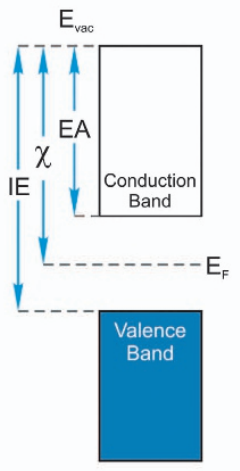

c

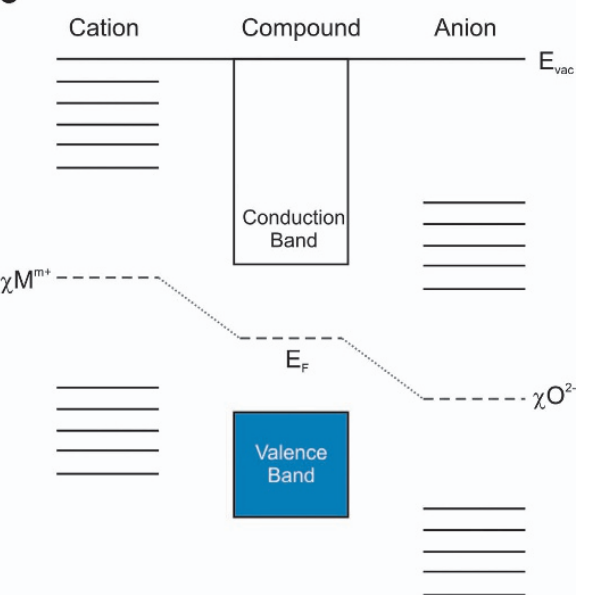

Figure 11 Illustrations of relationships between electronegativity $(\chi)$, ionization energy (IE), electron affinity (EA) and Fermi level ( $E_{\mathrm{F}}$ ). Schematic energylevel diagrams of (a) an isolated atom, (b) a condensed solid semiconductor and (c) a binary oxide (adapted from Campet et al. ${ }^{214}$ ). The electronegativity of an isolated metal cation is labeled $\chi \mathrm{M}^{m+}$, and an isolated oxygen anion is labeled $\chi \mathrm{O}^{2-}$.

a

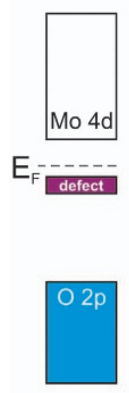

b

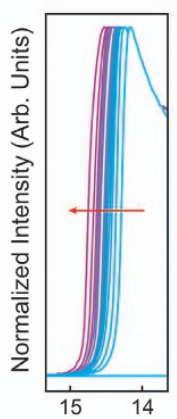

c

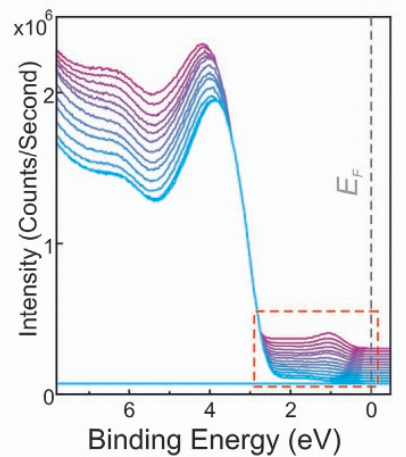

d

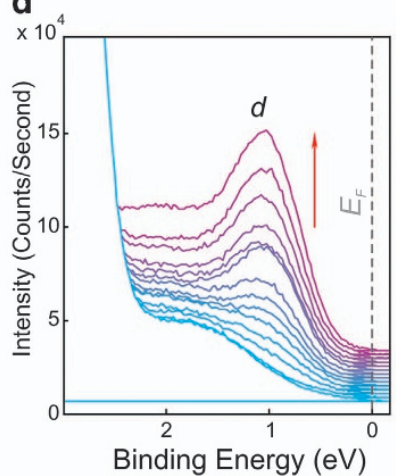

e

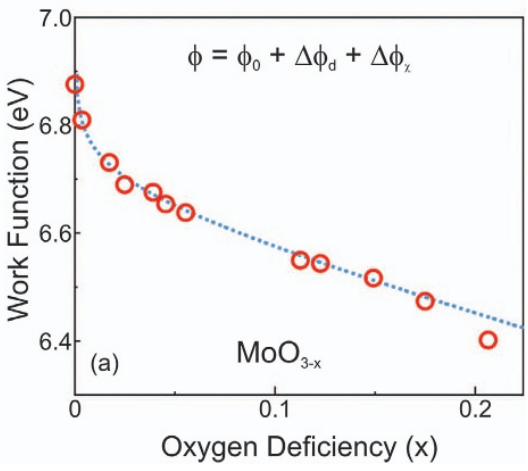

Figure 12 (a) Energy-level schematic of O-deficient $\mathrm{MoO}_{3}$, illustrating the positions of the Fermi level ( $E_{\mathrm{F}}$ ), $02 p$ band, Mo $4 d$ band and defect band. (b-d) Ultraviolet photoemission spectra of $\mathrm{MoO}_{3}$ as oxygen vacancies are introduced, including (b) secondary electron cut-off, (c) valence band and (d) an expanded view of the shallow valence features. The feature labeled ' $d$ ' is the gap state resulting from the oxygen vacancy defects. (e) Plot of work function versus oxygen deficiency, $x$. The red circles are experimental measurements, and the dashed blue curve is the predicted trend from dopant state $\left(\Delta \phi_{d}\right)$ and electronegativity $\left(\Delta \phi_{\chi}\right)$ arguments. 106

Metal oxides can exhibit a continual range of stoichiometries because they are prone to many different types of defect, such as oxygen vacancies, oxygen interstitials, cation vacancies and cation interstitials. Metal-oxide work functions can also be tuned over a continual range by changing the concentration of defects-removing oxygen tends to decrease the work function, whereas adding oxygen tends to increase the work function. Removal of oxygen through the formation of $\mathrm{O}$ vacancies results in cation centers with reduced oxidation states. As the concentration of reduced cations increases, an oxide's group electronegativity, and subsequently its work function, decreases. An example of the effect of O-vacancy defects on an oxide's work function is illustrated with $\mathrm{MoO}_{3}$ in Figure 12 .

In Figure 12, one can see that the increase in $\mathrm{O}$ vacancies gives rise to a decrease in work function (as seen by the shift of the secondary electron cut-off in Figure 12b). The O $2 p$ band also shifts to higher binding energy because the Fermi level moves closer to the conduction band as O-vacancy concentration increases (as seen in Figure 12c). Furthermore, as defect state appears within the band gap and increases in intensity as $\mathrm{O}$-vacancy concentration increases (as seen in Figure 12d).
In the presence of defects or dopants, the Fermi level is no longer positioned midway between the conduction band and the valence band, but rather it is shifted away from midgap position towards one of the band edges (depending on whether the defect/dopant is $n$ - or $p$-type). The electronegativity contribution to the Fermi level is an electrostatic argument that positions the valence and conduction bands relative to the vacuum level. The presence of dopants and defects can form additional occupied or unoccupied states within the band gap. These states alter the carrier concentrations (that is, holes or electrons in the valence band or conduction band, respectively) and consequently shift the Fermi level within the band gap. Thus, the Fermi-level position is the net result of electronegativity and carrier-concentration contributions.

As shown in Figure 12, oxygen vacancies in $\mathrm{MoO}_{3}$ give rise to an occupied donor state (seen at a binding energy of $c a .1 \mathrm{eV}$ in Figure 12d). There is a logarithmic relationship between donor concentration and Fermi-level position. ${ }^{215}$ Consequently, there is a rapid initial decrease in work function as oxygen vacancies are introduced, as seen in Figure 12e. The linear decrease in work function 
that occurs at higher O-vacancy concentrations is a consequence of electronegativity.

As mentioned in an earlier section, metal oxides exhibit doping asymmetry, ${ }^{191}$ meaning some oxides are prone to forming one type of defect over another. Because of their native defects, many oxides tend to have either $n$ - or $p$-type character. For instance, native $\mathrm{O}$ vacancies in oxides like $\mathrm{MoO}_{3}, \mathrm{TiO}_{2}, \mathrm{WO}_{3}$ and $\mathrm{V}_{2} \mathrm{O}_{5}$ make them 'intrinsic' $n$-type materials, whereas native $\mathrm{O}$ interstitials in oxides like $\mathrm{NiO}$ make them 'intrinsic' p-type semiconductors.

Based on these characteristics, one can develop strategies for tuning oxide properties for buffer layers. Decreasing an oxide's work function by inducing $\mathrm{O}$ vacancies may be a useful strategy for obtaining lowwork-function anode buffer layers, such as defective $\mathrm{ZrO}_{2}$. The opposite strategy (that is, inducing $\mathrm{O}$ interstitials) may be a useful strategy for obtaining high-work-function electrodes. Although, this may not work for $\mathrm{MoO}_{3}$, in which $\mathrm{O}$ interstitials are not stable, $\mathrm{NiO}$ supports $\mathrm{O}$ interstitials, and its work function has been reported to reach as high as $6.7 \mathrm{eV}$ by treating it in atmospheres of high oxygen potential. $^{216}$

\section{OPPORTUNITIES AND CHALLENGES OF METAL OXIDES AS ULTRATHIN BUFFER LAYERS}

In general, oxide buffer layers are very thin (within a few nanometers) and must be in physical contact with a highly conductive electrode. The electrode is often a metal or transparent conducting oxide. The interface with the electrode may have a chemical interaction with the buffer layer that extends several nanometers from the electrode/buffer interface. These interactions can influence the buffer layer's work function and electronic properties.

A number of studies have demonstrated the influence of oxide buffer-layer thickness on device performance. ${ }^{68,69,92,217}$ In general, it is found that there is an optimal thickness for a buffer layer. If the buffer layer is too thin or too thick, then device performance suffers. The optimal thickness of $\mathrm{MoO}_{3}$ has been found to be in the range of $0.5-3 \mathrm{~nm}$.

However, the optimal thickness of an oxide also depends on the nature of the electrode material. A number of studies have demonstrated chemical interactions between oxides and metal electrodeswithin the first few nanometers of an interface-and also dependence of device performance on the electrode material. ${ }^{66,70,218-222}$

Chemically reactive interfaces can give rise to reduction of oxidation state in the oxide buffer layer near the electrode/buffer interface. For instance, $\mathrm{MoO}_{3}$ on metals with various reactivity gives rise to reduced $\mathrm{MoO}_{3}$ near the metal/metal-oxide interface, as illustrated in Figure 13.

If the magnitude of the first oxidation potential of the metal electrode is greater than the first reduction potential of the oxide buffer layer, then the metal electrode will sequester oxygen anions from the oxide buffer layer. Thereby, the metal becomes oxidized and the oxide reduced, within the first few nanometers of the electrode/ buffer interface. The degree of oxidation/reduction is determined by the difference between the metal's first oxidation potential and the oxide's first reduction potential.

This results of such interfacial reactions causes changes to the oxide's properties near the electrode interface, and a strong dependence of a buffer layer's properties on its thickness. For instance, it has been demonstrated that interfacial reaction between $\mathrm{MoO}_{3}$ and various metal electrodes results in a decreased oxide work function and gap states close to the interface, as shown in Figure 14. Consequently, to ensure that an oxide buffer layer has the expected properties, one must ensure that there are no strong interfacial
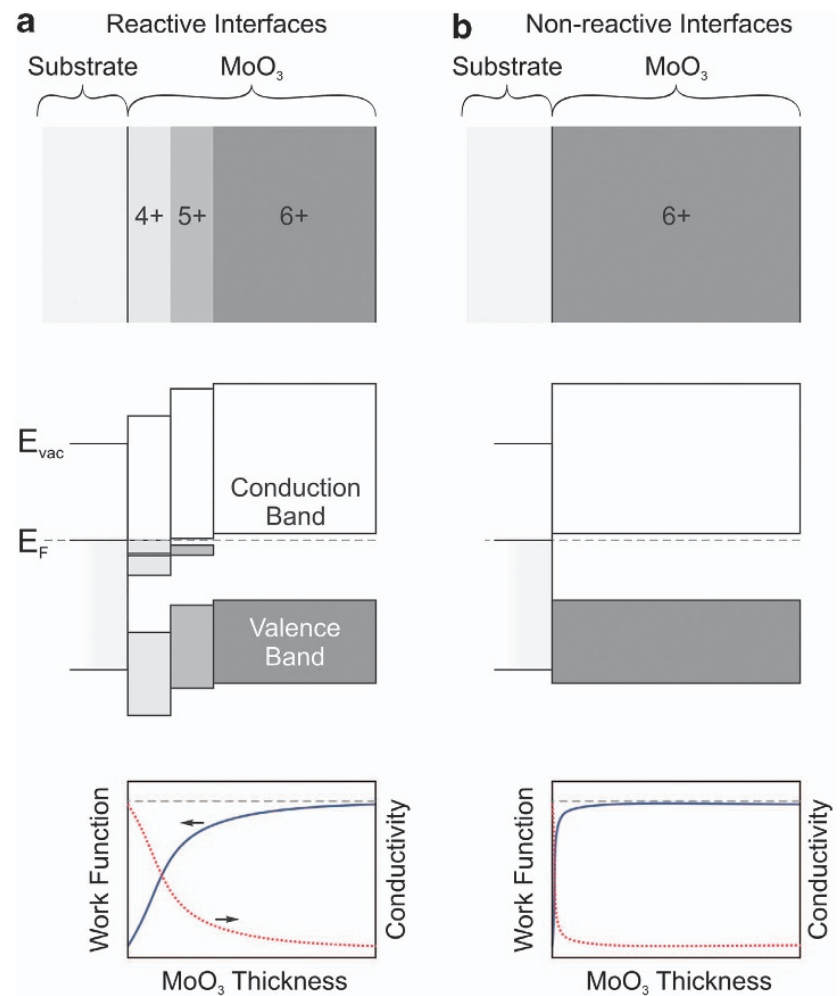

Figure 13 Illustration of the distribution of $\mathrm{Mo}$ oxidation state in ' $\mathrm{MoO}_{3}$ ' near an interface (top), schematic energy-band diagram for $\mathrm{MoO}_{3}$ near an interface (middle) and plot of work function versus $\mathrm{MoO}_{3}$ thickness and conductivity versus $\mathrm{MoO}_{3}$ thickness (bottom) for reactive (a) and nonreactive (b) interfaces between $\mathrm{MoO}_{3}$ and various electrode metals. ${ }^{224}$

reaction at the electrode/buffer interface or at least account for the interfacial reaction zone.

The degree of $\mathrm{MoO}_{3}$ reduction and the thickness required before $\mathrm{MoO}_{3}$ reaches its bulk properties depend on the metal electrode. As shown here, the metals that have greater first oxidation potentials than the first reduction potential of $\mathrm{MoO}_{3}$ (that is, $\mathrm{Ni}, \mathrm{Mo}$ and $\mathrm{V}$ ) cause $\mathrm{Mo}^{6+}$ cations to be reduced to $\mathrm{Mo}^{5+}$ (and $\mathrm{Mo}^{4+}$ in the case of V) close to the metal/oxide interface. Copper is unique in that it forms an oxide alloy with $\mathrm{MoO}_{3}$. Interestingly, slight $\mathrm{Mo}^{6+}$ reduction is also observed at the $\mathrm{Au} / \mathrm{MoO}_{3}$ interface; however, $\mathrm{Au}$ was not oxidized in the process. This suggests a charge-transfer interaction, possibly where $\mathrm{Mo}^{5+}$ cations are stabilized by screening from the Au Fermi level.

It has been shown on several occasions that organic ELA depends on buffer thickness. ${ }^{222,223}$ Based on the observed correlation between interfacial oxide reduction and oxide thickness, it appears that too thin of an oxide does not provide sufficient work function for favorable ELA (only in the case of high-work-function hole-injection buffer layers), whereas too thick of an oxide becomes too insulating and causes a high-series resistance in a device. Thus, a clear understanding of interfacial reduction reaction will provide a rational guide to process engineers to select best suitable electrode/ oxide structures for a targeted application.

\section{SUMMARY}

There are many types of buffer-layer materials that can be used in organic electronic devices. Among the most versatile are transition metal oxides. These materials can be used in OLEDs, OPVs and 
a

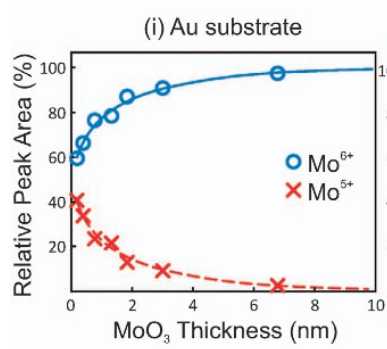

(ii) Ni substrate

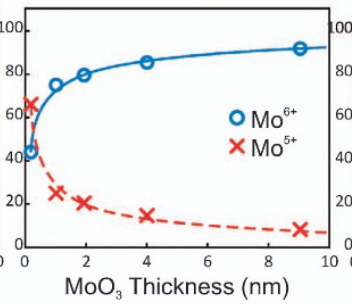

Oxidation-State Profiles

(iii) Mo substrate

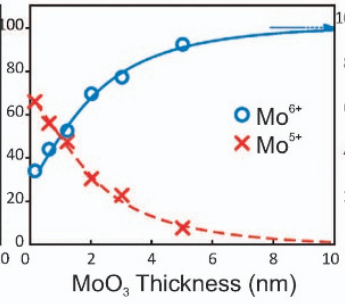

(iv) V substrate

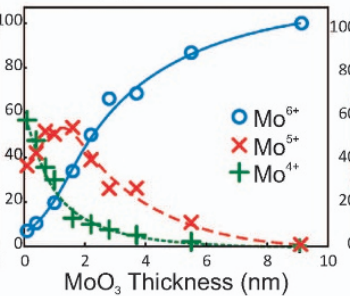

(v) Cu substrate

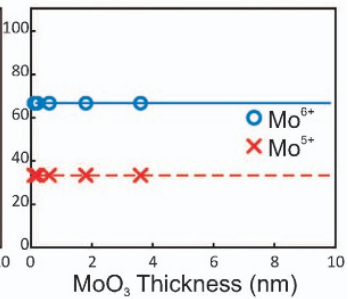

b

Work-Function Profiles

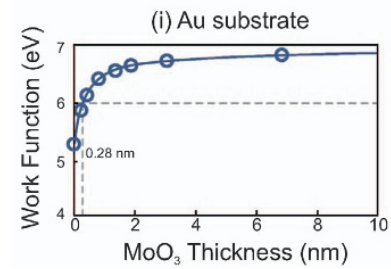

(ii) Ni substrate

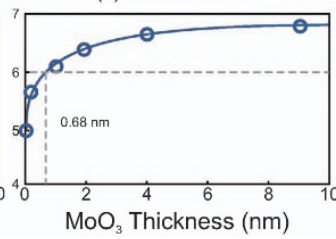

(iii) Mo substrate

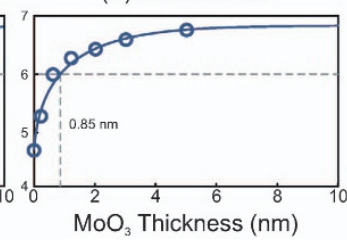

(iv) $\mathrm{V}$ substrate

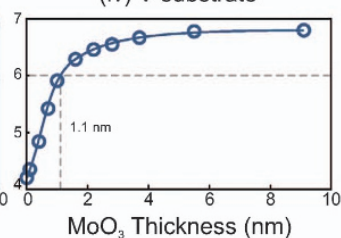

(v) Cu substrate

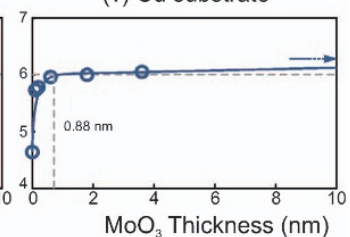

Figure 14 (a) Oxidation state profiles and (b) work-function profiles for $\mathrm{MoO}_{3}$ films grown on various metal electrodes. Chemical/charge-transfer interactions between the electrode metals and the oxide buffer layer lead to oxide cation reduction and decreased work function within the first few nanometers of the metal/oxide interface. ${ }^{224}$

OTFTs as both electron-injection and hole-injection contacts. Because of the weakly interacting nature of the metal-oxide/organic semiconductor interface, such interfaces follow a general ELA trend, in which hole-injection and electron-injection barriers are governed by an organic molecule's HOMO/LUMO levels and an electrode's work function. Transition metal oxides exhibit a wide range of work functions that can be altered by changing the concentration of defects and the cation oxidation states. Generally, decreasing the cation oxidation state gives a decrease in work function, due to the lower electronegativity of low-oxidation-state cations. Defects and changes to oxidation state also result in changes to an oxide's electronic structure. An oxide's electronic structure can influence the rectifying character of a buffer layer. Such layers can be designed such that they allow injection of one type of charge charier in one direction, but suppress movement of charge in the opposite direction. Although such buffer layers can be very useful, one must be mindful of the contact between the buffer layer and the electrode. Interfacial oxidation/reduction reactions can give rise to changes in an oxide buffer layer's stoichiometry, electronic structure and work function nearby electrode/oxide interfaces. These changes can affect the oxide buffer layer's ability to provide ELA and charge-selective contacts.

1 Shaheen, S. E., Brabec, C. J., Sariciftci, N. S., Padinger, F., Fromherz, T. \& Hummelen, J. C. $2.5 \%$ Efficient organic plastic solar cells. Appl. Phys. Lett. 78, 841-843 (2001).

2 He, Z., Zhong, C., Su, S., Xu, M., Wu, H. \& Cao, Y. Enhanced power-conversion efficiency in polymer solar cells using an inverted device structure. Nat. Photon 6, 591-595 (2012).

3 Sekitani, T., Zschieschang, U., Klauk, H. \& Someya, T. Flexible organic transistors and circuits with extreme bending stability. Nat. Mater. 9, 1015-1022 (2010).

4 Meijer, E. J., de Leeuw, D. M., Setayesh, S., van Veenendaal, E., Huisman, B. H., Blom, P. W. M., Hummelen, J. C., Scherf, U. \& Klapwijk, T. M. Solution-processed ambipolar organic field-effect transistors and inverters. Nat. Mater. 2, 678-682 (2003).

5 McCarthy, M. A., Liu, B., Donoghue, E. P., Kravchenko, I., Kim, D. Y., So, F. \& Rinzler, A. G. Low-voltage, low-power, organic light-emitting transistors for active matrix displays. Science 332, 570-573 (2011)

6 Yoo, B., Jones, B. A., Basu, D., Fine, D., Jung, T., Mohapatra, S., Facchetti, A., Dimmler, K., Wasielewski, M. R., Marks, T. J. \& Dodabalapur, A. High-performance solution-deposited n-channel organic transistors and their complementary circuits. Adv. Mater. 19, 4028-4032 (2007).
7 Singh, T. B. \& Sariciftci, N. S. Progress in plastic electronics devices. Annu. Rev. Mater. Res. 36, 199-230 (2006).

8 Briseno, A. L., Kim, F. S., Babel, A., Xia, Y. \& Jenekhe, S. A. N-channel polymer thin film transistors with long-term air-stability and durability and their use in complementary inverters. J. Mater. Chem. 21, 16461-16466 (2011).

9 Clemens, W., Fix, I., Ficker, J., Knobloch, A. \& Ullmann, A. From polymer transistors toward printed electronics. J. Mater. Res. 19, 1963-1973 (2004).

10 Klauk, H., Zschieschang, U., Pflaum, J. \& Halik, M. Ultralow-power organic complementary circuits. Nature 445, 745-748 (2007).

11 Ishii, H., Sugiyama, K., Ito, E. \& Seki, K. Energy level alignment and interfacial electronic structures at organic metal and organic organic interfaces. Adv. Mater. 11, 605-625 (1999).

12 Hung, L. S., Tang, C. W. \& Mason, M. G. Enhanced electron injection in organic electroluminescence devices using an Al/LiF electrode. Appl. Phys. Lett. 70, 152-154 (1997).

13 Koch, N. Organic electronic devices and their functional interfaces. Chemphyschem 8, 1438-1455 (2007).

14 Scott, J. C. Metal-organic interface and charge injection in organic electronic devices. J. Vacuum Sci. Technol. A 21, 521-531 (2003).

15 Blyth, R. I. R., Duschek, R., Koller, G., Netzer, F. P. \& Ramsey, M. G. Band alignment in organic devices: photoemission studies of model oligomers on $\ln _{2} \mathrm{O}_{3}$. J. Appl. Phys. 90, 270-275 (2001).

16 Zhang, Y. Q., Acharya, R. \& Cao, X. A. Efficient and reliable green organic light emitting diodes with $\mathrm{cl}_{2}$ plasma-etched indium tin oxide anode. J. Appl. Phys. 112, 013103 (2012)

17 Xu, Z. Q., Li, J., Yang, J. P., Cheng, P. P., Zhao, J., Lee, S. T., Li, Y. Q. \& Tang, J. X. Enhanced performance in polymer photovoltaic cells with chloroform treated indium tin oxide anode modification. Appl. Phys. Lett. 98, 253303 (2011).

18 Helander, M. G., Wang, Z. B., Qiu, J., Greiner, M. T., Puzzo, D. P., Liu, Z. W. \& Lu, Z. $\mathrm{H}$. Chlorinated indium tin oxide electrodes with high work function for organic device compatibility. Science 332, 944-947 (2011).

19 Sun, K. \& Ouyang, J. Polymer solar cells using chlorinated indium tin oxide electrodes with high work function as the anode. Solar Energy Mater. Solar Cells 96, 238-243 (2012).

20 Wang, Q., Williams, G. \& Aziz, H. Photo-degradation of the indium tin oxide (Ito)/organic interface in organic optoelectronic devices and a new outlook on the role of ITO surface treatments and interfacial layers in improving device stability. Org. Electron. 13, 2075-2082 (2012).

21 Wu, C. C., Wu, C. I., Sturm, J. C. \& Kahn, A. Surface modification of indium tin oxide by plasma treatment: an effective method to improve the efficiency, brightness, and reliability of organic light emitting devices. Appl. Phys. Lett. 70, 1348-1350 (1997).

22 Lu, D., Wu, Y., Guo, J., Lu, G., Wang, Y. \& Shen, J. Surface treatment of indium tin oxide by oxygen-plasma for organic light-emitting diodes. Mate. Sci. Eng. B 97, 141-144 (2003).

23 Ding, X. M., Hung, L. M., Cheng, L. F., Deng, Z. B., Hou, X. Y., Lee, C. S. \& Lee, S. T. Modification of the hole injection barrier in organic light-emitting devices studied by ultraviolet photoelectron spectroscopy. Appl. Phys. Lett. 76, 2704-2706 (2000).

24 Kim, S. Y., Lee, J. L., Kim, K. B. \& Tak, Y. H. Effect of ultraviolet-ozone treatment of indium-tin-oxide on electrical properties of organic light emitting diodes. J. Appl. Phys. 95, 2560-2563 (2004). 
25 Sugiyama, K., Ishii, H., Ouchi, Y. \& Seki, K. Dependence of indium-tin-oxide work function on surface cleaning method as studied by ultraviolet and X-ray photoemission spectroscopies. J. Appl. Phys. 87, 295-298 (2000).

26 Irfan, I., Graber, S., So, F. \& Gao, Y. Interplay of cleaning and de-doping in oxygen plasma treated high work function indium tin oxide. Org. Electron. 13, 2028-2034 (2012).

27 Jung, M. -H. \& Choi, H. -S. Surface treatment and characterization of ito thin films using atmospheric pressure plasma for organic light emitting diodes. J. Colloid. Interface. Sci. 310, 550-558 (2007)

28 Mason, M. G., Hung, L. S., Tang, C. W., Lee, S. T., Wong, K. W. \& Wang, M. Characterization of treated indium-tin-oxide surfaces used in electroluminescent devices. J. Appl. Phys. 86, 1688-1692 (1999)

29 Helander, M. G., Wang, Z. B., Greiner, M. T., Liu, Z. W., Qiu, J. \& Lu, Z. H. Oxidized gold thin films: an effective material for high-performance flexible organic optoelectronics. Adv. Mater. 22, 2037-2040 (2010).

30 Li, C. N., Kwong, C. Y., Djurišić, A. B., Lai, P. T., Chui, P. C., Chan, W. K. \& Liu, S. Y. Improved performance of OLEDs with ITO surface treatments. Thin Solid Films 477 , 57-62 (2005)

31 Irfan, I. \& Gao, Y. Effects of exposure and air annealing on $\mathrm{MoO}_{\mathrm{x}}$ thin films. J. Photonics Energy 2, 021213 (2012).

32 Irfan, I., Ding, H., Gao, Y., Small, C., Kim, D. Y., Subbiah, J. \& So, F. Energy level evolution of air and oxygen exposed molybdenum trioxide films. Appl. Phys. Lett. 96, 243307 (2010).

33 Wan, A., Hwang, J., Amy, F. \& Kahn, A. Impact of electrode contamination on the alpha-NPD/Au hole injection barrier. Org. Electron. 6, 47-54 (2005)

34 Meyer, J., Shu, A., Kroger, M. \& Kahn, A. Effect of contamination on the electronic structure and hole-injection properties of $\mathrm{MoO}_{3}$ /organic semiconductor interfaces. Appl. Phys. Lett. 96, 133308 (2010).

35 Cheung, C. H., Song, W. J. \& So, S. K. Role of air exposure in the improvement of injection efficiency of transition metal oxide/organic contact. Org. Electron. 11, 89-94 (2010).

36 Grobosch, M. \& Knupfer, M. Charge-injection barriers at realistic metal/organic interfaces: metals become faceless. Adv. Mater. 19, 754-756 (2007).

37 VanSlyke, S. A., Chen, C. H. \& Tang, C. W. Organic electroluminescent devices with improved stability. Appl. Phys. Lett. 69, 2160-2162 (1996).

38 Shirota, Y., Kuwabara, Y., Inada, H., Wakimoto, T., Nakada, H., Yonemoto, Y., Kawami, S. \& Imai, K. Multilayered organic electroluminescent device using a nove starburst molecule, 4,4',4'-Tris(3-methylphenylphenylamino)triphenylamine, as a hole transport material. Appl. Phys. Lett. 65, 807-809 (1994).

39 Hughes, G. \& Bryce, M. R. Electron-transporting materials for organic electroluminescent and electrophosphorescent devices. J. Mater. Chem. 15, 94-107 (2005).

$40 \mathrm{Ma}$, H., Yip, H. L., Huang, F. \& Jen, A. K. Y. Interface engineering for organic electronics. Adv. Funct. Mater. 20, 1371-1388 (2010).

41 Zhou, Y. H., Fuentes-Hernandez, C., Shim, J., Meyer, J., Giordano, A. J., Li, H. Winget, P., Papadopoulos, T., Cheun, H., Kim, J., Fenoll, M., Dindar, A., Haske, W., Najafabadi, E., Khan, T. M., Sojoudi, H., Barlow, S., Graham, S., Bredas, J. L., Marder, S. R., Kahn, A. \& Kippelen, B. A universal method to produce low-work function electrodes for organic electronics. Science 336, 327-332 (2012).

42 Ratcliff, E. L., Lee, P. A. \& Armstrong, N. R. Work function control of hole-selective polymer/ito anode contacts: an electrochemical doping study. J. Mater. Chem. 20, 2672-2679 (2010).

43 Brown, T. M., Kim, J. S., Friend, R. H., Cacialli, F., Daik, R. \& Feast, W. J. Built-in field electroabsorption spectroscopy of polymer light-emitting diodes incorporating a doped poly(3,4-ethylene dioxythiophene) hole injection layer. Appl. Phys. Lett. 75, 1679-1681 (1999).

44 Huang, F., Niu, Y. H., Zhang, Y., Ka, J. W., Liu, M. S. \& Jen, A. K. Y. A conjugated, neutral surfactant as electron-injection material for high-efficiency polymer lightemitting diodes. Adv. Mater. 19, 2010-2014 (2007).

45 Oh, S. - H., Vak, D., Na, S. -I., Lee, T. -W. \& Kim, D. -Y. Water-soluble polyfluorenes as an electron injecting layer in pleds for extremely high quantum efficiency. Adv. Mater. 20, 1624-1629 (2008)

46 Ma, W. L., Iyer, P. K., Gong, X., Liu, B., Moses, D., Bazan, G. C. \& Heeger, A. J. Water/ methanol-soluble conjugated copolymer as an electron-transport layer in polymer light-emitting diodes. Adv. Mater. 17, 274-277 (2005).

47 Jabbour, G. E., Kippelen, B., Armstrong, N. R. \& Peyghambarian, N. Aluminum based cathode structure for enhanced electron injection in electroluminescent organic devices. Appl. Phys. Lett. 73, 1185-1187 (1998)

48 Lee, J., Park, Y., Kim, D. Y., Chu, H. Y., Lee, H. \& Do, L. M. High efficiency organic light-emitting devices with Al/NaF cathode. Appl. Phys. Lett. 82, 173-175 (2003).

49 Park, Y., Lee, J., Lee, S. K. \& Kim, D. Y. Photoelectron spectroscopy study of the electronic structures of $\mathrm{Al} / \mathrm{MgF}_{2} /$ Tris-(8-Hydroxyquinoline) aluminum interfaces. Appl. Phys. Lett. 79, 105-107 (2001)

50 Lee, J., Park, Y., Lee, S. K., Cho, E. J., Kim, D. Y., Chu, H. Y., Lee, H., Do, L. M. \& Zyung, T. Tris-(8-hydroxyquinoline)aluminum-based organic light-emitting devices with $\mathrm{Al} / \mathrm{CaF}_{2}$ cathode: performance enhancement and interface electronic structures. Appl. Phys. Lett. 80, 3123-3125 (2002)

51 Chen, M. H. \& Wu, C. I. The roles of thermally evaporated cesium carbonate to enhance the electron injection in organic light emitting devices. J. Appl. Phys. 104, 113713 (2008).

52 Huang, J., Xu, Z. \& Yang, Y. Low-work-function surface formed by solution-processed and thermally deposited nanoscale layers of cesium carbonate. Adv. Funct. Mater. $17,1966-1973$ (2007)
53 Khodabakhsh, S., Poplavskyy, D., Heutz, S., Nelson, J., Bradley, D. D. C., Murata, F. \& Jones, T. S. Using self-assembling dipole molecules to improve hole injection in conjugated polymers. Adv. Funct. Mater. 14, 1205-1210 (2004).

54 Huang, Q. L., Evmenenko, G. A., Dutta, P., Lee, P., Armstrong, N. R. \& Marks, T. J. Covalently bound hole-injecting nanostructures. systematics of molecular architecture, thickness, saturation, and electron-blocking characteristics on organic light-emitting diode luminance, turn-on voltage, and quantum efficiency. J. Am. Chem. Soc. 127, 10227-10242 (2005).

55 Lee, J., Jung, B. J., Lee, J. I., Chu, H. Y., Do, L. M. \& Shim, H. K. Modification of an ITO anode with a hole-transporting sam for improved OLED device characteristics. J. Mater. Chem. 12, 3494-3498 (2002).

56 Hatton, R. A., Day, S. R., Chesters, M. A. \& Willis, M. R. Organic electroluminescent devices: enhanced carrier injection using an organosilane self assembled monolayer derivatized ITO electrode. Thin Solid Films 394, 292-297 (2001).

57 Bardecker, J. A., Ma, H., Kim, T., Huang, F., Liu, M. S., Cheng, Y. J., Ting, G. \& Jen, A. K. Y. Self-assembled electroactive phosphonic acids on ito: maximizing hole-injection in polymer light-emitting diodes. Adv. Funct. Mater. 18, 3964-3971 (2008).

58 Marmont, P., Battaglini, N., Lang, P., Horowitz, G., Hwang, J., Kahn, A., Amato, C. \& Calas, P. Improving charge injection in organic thin-film transistors with thiol-based self-assembled monolayers. Org. Electron. 9, 419-424 (2008).

59 Lee, H., Lee, J., Jeon, P., Jeong, K., Yi, Y., Kim, T. G., Kim, J. W. \& Lee, J. W. Highly enhanced electron injection in organic light-emitting diodes with an N-type semiconducting $\mathrm{MnO}_{2}$ layer. Org. Electron. 13, 820-825 (2012).

60 Qiu, C. F., Xie, Z. L., Chen, H. Y., Wong, M. \& Kwok, H. S. Comparative study of metal or oxide capped indium-tin oxide anodes for organic light-emitting diodes. J. Appl. Phys. 93, 3253-3258 (2003).

61 Tokito, S., Noda, K. \& Taga, Y. Metal oxides as a hole-injecting layer for an organic electroluminescent device. J. Phys. D Appl. Phys. 29, 2750-2753 (1996).

62 Xie, G. H., Meng, Y. L., Wu, F. M., Tao, C., Zhang, D. D., Liu, M. J., Xue, Q., Chen, W. \& Zhao, Y. Very low turn-on voltage and high brightness Tris-(8-hydroxyquinoline) aluminum-based organic light-emitting diodes with a $\mathrm{MoO}_{\mathrm{x}}$ p-doping layer. Appl. Phys. Lett. 92, 093305 (2008).

63 Wang, F. X., Qiao, X. F., Xiong, T. \& Ma, D. G. The role of molybdenum oxide as anode interfacial modification in the improvement of efficiency and stability in organic lightemitting diodes. Org. Electron. 9, 985-993 (2008).

64 Kroger, M., Hamwi, S., Meyer, J., Riedl, T., Kowalsky, W. \& Kahn, A. Role of the deeplying electronic states of $\mathrm{MoO}_{3}$ in the enhancement of hole-injection in organic thin films. Appl. Phys. Lett. 95, 123301 (2009).

65 Hamwi, S., Meyer, J., Winkler, T., Riedl, T. \& Kowalsky, W. p-Type doping efficiency of $\mathrm{MoO}_{3}$ in organic hole transport materials. Appl. Phys. Lett. 94, 253307 (2009).

66 Lee, H., Cho, S. W., Han, K., Jeon, P. E., Whang, C. N., Jeong, K., Cho, K. \& Yi, Y. The origin of the hole injection improvements at indium tin oxide/molybdenum trioxide/ $\mathrm{N}, \mathrm{N}^{\prime}$-bis(1-naphthyl)-N,N'-diphenyl-1,1'-biphenyl-4,4'-diamine interfaces. Appl. Phys. Lett. 93, 043308 (2008).

67 Matsushima, T., Jin, G. H. \& Murata, H. Marked improvement in electroluminescence characteristics of organic light-emitting diodes using an ultrathin hole-injection layer of molybdenum oxide. J. Appl. Phys. 104, 054501 (2008).

68 Matsushima, T. \& Murata, H. Observation of space-charge-limited current due to charge generation at interface of molybdenum dioxide and organic layer. Appl. Phys. Lett. 95, 203306 (2009).

69 Matsushima, T., Kinoshita, Y. \& Murata, H. Formation of ohmic hole injection by inserting an ultrathin layer of molybdenum trioxide between indium tin oxide and organic hole-transporting layers. Appl. Phys. Lett. 91, 253504 (2007).

70 Yi, Y., Jeon, P. E., Lee, H., Han, K., Kim, H. S., Jeong, K. \& Cho, S. W. The interface state assisted charge transport at the $\mathrm{MoO}_{3} /$ metal interface. J. Chem. Phys. 130 (2009)

71 Yook, K. S. \& Lee, J. Y. Low driving voltage in organic light-emitting diodes using $\mathrm{MoO}_{3}$ as an interlayer in hole transport layer. Synth. Met. 159, 69-71 (2009).

72 Wang, Z. B., Helander, M. G., Greiner, M. T., Qiu, J. \& Lu, Z. H. Analysis of chargeinjection characteristics at electrode-organic interfaces: case study of transitionmetal oxides. Phys. Rev. B. 80, 235325 (2009)

73 Meyer, J., Winkler, T., Hamwi, S., Schmale, S., Johannes, H. H., Weimann, T., Hinze, P., Kowlasky, W. \& Riedl, T. Transparent inverted organic light-emitting diodes with a tungsten oxide buffer layer. Adv. Mater. 20, 3839-3843 (2008).

74 Park, S. W., Choi, J. M., Kim, E. \& Im, S. Inverted top-emitting organic light-emitting diodes using transparent conductive $\mathrm{NiO}$ electrode. Appl. Surf. Sci. 244, 439-443 (2005)

75 Woo, S., Kim, J., Cho, G., Kim, K., Lyu, H. \& Kim, Y. Influence of nickel oxide nanolayer and doping in organic light-emitting devices. J. Ind. Eng. Chem. 15, 716-718 (2009).

76 Chan, I. M. Hsu, T. Y. \& Hong, F. C. Enhanced hole injections in organic lightemitting devices by depositing nickel oxide on indium tin oxide anode. Appl. Phys. Lett. 81, 1899-1901 (2002).

77 Chun, J. Y., Han, J. W. \& Seo, D. S. Application of High work function anode for organic light emitting diode. Mol. Cryst. Liq. Cryst. 514, 445-451 (2009).

78 Wei, B., Yamamoto, S., Ichikawa, M., Li, C., Fukuda, T. \& Taniguchi, Y. High-efficiency transparent organic light-emitting diode with one thin layer of nickel oxide on a transparent anode for see-through-display application. Semiconductor Sci. Technol. 22, 788-792 (2007).

79 Im, H. C., Choo, D. C., Kim, T. W., Kim, J. H., Seo, J. H. \& Kim, Y. K. Highly efficient organic light-emitting diodes fabricated utilizing nickel-oxide buffer layers between the anodes and the hole transport layers. Thin Solid Films $\mathbf{5 1 5}$ 5099-5102 (2007) 
80 Steirer, K. X., Chesin, J. P., Widjonarko, N. E., Berry, J. J., Miedaner, A., Ginley, D. S \& Olson, D. C. Solution deposited nio thin-films as hole transport layers in organic photovoltaics. Org. Electron. 11, 1414-1418 (2010).

81 Irwin, M. D., Buchholz, B., Hains, A. W., Chang, R. P. H. \& Marks, T. J. p-Type semiconducting nickel oxide as an efficiency-enhancing anode interfacia layer in polymer bulk-heterojunction solar cells. Proc. Natl Acad. Sci. USA 105, 2783-2787 (2008)

82 Wang, S. A., Osasa, T. \& Matsumura, M. $\mathrm{CuO}_{x}$ films as anodes for organic lightemitting devices. Jpn. J. Appl. Phys. Part 1-Regular Papers Brief Commun. Rev. Papers 45, 8894-8896 (2006).

83 Murdoch, G. B., Greiner, M., Helander, M. G., Wang, Z. B. \& Lu, Z. H. A comparison of $\mathrm{CuO}$ and $\mathrm{Cu}_{2} \mathrm{O}$ hole-injection layers for low voltage organic devices. Appl. Phys. Lett. 93, 083309 (2008).

84 Zhang, H. M. \& Choy, W. C. H. Highly efficient organic light-emitting devices with surface-modified metal anode by vanadium pentoxide. J Phys. D Appl. Phys. 41 062003 (2008)

85 Zhu, X. L., Sun, J. X., Yu, X. M., Wong, M. \& Kwok, H. S. Investigation of Al- and Agbased top-emitting organic light-emitting diodes with metal oxides as hole-injection layer. Jpn. J. Appl. Phys. Part 1-Regular Papers Brief Commun. Rev. Papers 46, 1033-1036 (2007).

86 Meyer, J., Zilberberg, K., Riedl, T. \& Kahn, A. Electronic structure of vanadium pentoxide: an efficient hole injector for organic electronic materials. J. Appl. Phys 110, 033710 (2011).

87 Zhang, D. D., Feng, J., Liu, Y. F., Zhong, Y. Q., Bai, Y., Jin, Y., Xie, G. H., Xue, Q., Zhao, Y., Liu, S. Y. \& Sun, H. B. Enhanced hole injection in organic light-emitting devices by using $\mathrm{Fe}_{3} \mathrm{O}_{4}$ as an anodic buffer layer. Appl. Phys. Lett. 94, 223306 (2009).

88 Xiao, B. W., Shang, Y. F., Meng, M. \& Li, C. N. Enhancement of hole injection with an ultra-thin $\mathrm{Ag}_{2} \mathrm{O}$ modified anode in organic light-emitting diodes. Microelectron. J. 36, 105-108 (2005).

89 Bolink, H. J., Coronado, E., Repetto, D., Sessolo, M., Barea, E. M., Bisquert, J., Garcia-Belmonte, G., Prochazka, J. \& Kavan, L. Inverted solution processable OLEDs using a metal oxide as an electron injection contact. Adv. Funct. Mater. 18, 145-150 (2008).

90 Bolink, H. J., Coronado, E., Repetto, D. \& Sessolo, M. Air stable hybrid organic inorganic light emitting diodes Using Zno as the cathode. Appl. Phys. Lett. 91, 223501 (2007)

91 Tokmoldin, N., Griffiths, N., Bradley, D. D. C. \& Haque, S. A. A hybrid inorganic organic semiconductor light-emitting diode using $\mathrm{ZrO}_{2}$ as an electron-injection layer. Adv. Mater. 21, 3475-3478 (2009).

92 Shrotriya, V., Li, G., Yao, Y., Chu, C. W. \& Yang, Y. Transition metal oxides as the buffer layer for polymer photovoltaic cells. Appl. Phys. Lett. 88, 073508 (2006).

93 Kim, J. Y., Lee, K., Coates, N. E., Moses, D., Nguyen, T. Q., Dante, M. \& Heeger, A. J. Efficient tandem polymer solar cells fabricated by all-solution processing. Science 317, 222-225 (2007).

94 Hancox, I., Rochford, L. A., Clare, D., Sullivan, P. \& Jones, T. S. Utilizing n-type vanadium oxide films as hole-extracting layers for small molecule organic photovoltaics. Appl. Phys. Lett. 99, 013304 (2011).

95 Tan, Z. a., Li, L., Cui, C., Ding, Y., Xu, Q., Li, S., Qian, D. \& Li, Y. Solution-processed tungsten oxide as an effective anode buffer layer for high-performance polymer sola cells. J. Phys. Chem. C 116, 18626-18632 (2012).

96 Tan, Z., Zhang, W., Cui, C., Ding, Y., Qian, D., Xu, Q., Li, L., Li, S. \& Li, Y. Solutionprocessed vanadium oxide as a hole collection layer on an ito electrode for highperformance polymer solar cells. Phys. Chem. Chem. Phys. 14, 14589-14595 (2012)

97 Chen, S., Manders, J. R., Tsang, S. -W. \& So, F. Metal oxides for interface engineering in polymer solar cells. J. Mater. Chem. 22, 24202-24212 (2012).

98 Nie, G., Peng, J., Lan, L., Xu, R., Zou, J. \& Cao, Y. Tuning on threshold voltage of organic field-effect transistor with a copper oxide layer. Org. Electron. 12, 429-434 (2011)

99 Darmawan, P., Minari, T., Kumatani, A., Li, Y., Liu, C. \& Tsukagoshi, K. Reduction of charge injection barrier by $1-\mathrm{nm}$ contact oxide interlayer in organic field effect transistors. Appl. Phys. Lett. 100, 013303 (2012).

$100 \mathrm{Yu}, \mathrm{X}$., Yu, J., Zhou, J. \& Jiang, Y. Organic field-effect transistors with nearly noninjection barrier from source/drain electrodes to pentacene. Synth. Met. 162, 936-940 (2012).

101 Kumaki, D., Umeda, T. \& Tokito, S. Reducing the contact resistance of bottom contact pentacene thin-film transistors by employing a moox carrier injection layer. Appl. Phys. Lett. 92, 013301 (2008).

102 Chu, C. W., Li, S. H., Chen, C. W., Shrotriya, V. \& Yang, Y. High-performance organic thin-film transistors with metal oxide/metal bilayer electrode. Appl. Phys. Lett. 87, 193508 (2005)

103 Yao, Z. Q., Liu, S. L., Zhang, L., He, B., Kumar, A., Jiang, X., Zhang, W. J. \& Shao, G. Room temperature fabrication of $\mathrm{p}$-channel $\mathrm{Cu}_{2} \mathrm{O}$ thin-film transistors on flexible polyethylene terephthalate substrates. Appl. Phys. Lett. 101, 042114 (2012).

104 Gwinner, M. C., Vaynzof, Y., Banger, K. K., Ho, P. K. H., Friend, R. H. \& Sirringhaus, $\mathrm{H}$. Solution-processed zinc oxide as high-performance air-stable electron injector in organic ambipolar light-emitting field-effect transistors. Adv. Funct. Mater. 20, 3457-3465 (2010)

105 Wang, Z. B., Helander, M. G., Qiu, J., Puzzo, D. P., Greiner, M. T., Hudson, Z. M., Wang, S., Liu, Z. W. \& Lu, Z. H. Unlocking the full potential of organic light-emitting diodes on flexible plastic. Nat. Photon 5, $753-757$ (2011).
106 Greiner, M. T., Chai, L., Helander, M. G., Tang, W. M. \& Lu, Z. H. Transition meta oxide work functions: the influence of cation oxidation state and oxygen vacancies. Adv. Funct. Mater. 22, 4557-4568 (2012).

107 Li, J. Z., Yahiro, M., Ishida, K., Yamada, H. \& Matsushige, K. Enhanced performance of organic light emitting device by insertion of conducting/insulating $\mathrm{WO}_{3}$ anodic buffer layer. Synth. Met. 151, 141-146 (2005).

108 Thimsen, E., Martinson, A. B. F., Elam, J. W. \& Pellin, M. J. Energy levels, electronic properties, and rectification in ultrathin p-nio films synthesized by atomic layer deposition. J. Phys. Chem. C 116, 16830-16840 (2012).

109 Buwen, X., Yafeng, S., Meng, M. \& Chuannan, L. Enhancement of hole injection with an ultra-thin $\mathrm{Ag}_{2} \mathrm{O}$ modified anode in organic light-emitting diodes. Microelec tron. J. 36, 105-108 (2005).

110 Lee, H., Park, L., Kwak, J., Yoon, D. Y \& Lee, C. Improvement of electron injection in inverted bottom-emission blue phosphorescent organic light emitting diodes using zinc oxide nanoparticles. Appl. Phys. Lett. 96, 153306 (2010).

111 Vasilopoulou, M., Kennou, S., Ladas, S., Georga, S. N., Botzakaki, M., Skarlatos, D. Krontiras, C. A., Stathopoulos, N. A., Argitis, P. \& Palilis, L. C. Atomic layer deposited zirconium oxide electron injection layer for efficient organic light emitting diodes. Org. Electron. 14, 312-319 (2013).

112 Ratcliff, E. L., Meyer, J., Steirer, K. X., Garcia, A., Berry, J. J., Ginley, D. S., Olson, D. C. Kahn, A. \& Armstrong, N. R. Evidence for near-surface $\mathrm{NiOOH}$ species in solutionprocessed nio ${ }_{x}$ selective interlayer materials: impact on energetics and the performance of polymer bulk heterojunction photovoltaics. Chem. Mater. 23, 4988-5000, 2011)

113 Zilberberg, K., Trost, S., Schmidt, H. \& Riedl, T. Solution processed vanadium pentoxide as charge extraction layer for organic solar cells. Adv. Energy Mater. 1, 377-381 (2011).

114 Huang, Z. H., Zeng, X. T., Kang, E. T., Fuh, Y. H. \& Lu, L. Ultrathin sol-gel titanium oxide hole injection layer in OLEDs. Surface Coatings Technol. 198, 357-361 (2005).

115 Puetz, A., Steiner, F., Mescher, J., Reinhard, M., Christ, N., Kutsarov, D., Kalt, H., Lemmer, U. \& Colsmann, A. Solution processable, precursor based zinc oxide buffer layers for $4.5 \%$ efficient organic tandem solar cells. Org. Electron. 13, 2696-2701 (2012).

116 de Bruyn, P., Moet, D. J. D. \& Blom, P. W. M. All-solution processed polymer lightemitting diodes with air stable metal-oxide electrodes. Org. Electron. 13, 1023-1030 (2012).

117 Meyer, J., Khalandovsky, R., Gorrn, P. \& Kahn, A. $\mathrm{MoO}_{3}$ films spin-coated from a nanoparticle suspension for efficient hole-injection in organic electronics. Adv. Mater. 23, 70-73 (2011).

118 Jasieniak, J. J., Seifter, J., Jo, J., Mates, T., Heeger, A. J. \& Solution-Processed MoOx, A. Anode interlayer for use within organic photovoltaic devices. Adv. Funct. Mater. 22 2594-2605 (2012).

119 Vasilopoulou, M., Papadimitropoulos, G., Palilis, L. C., Georgiadou, D. G., Argitis, P., Kennou, S., Kostis, I., Vourdas, N., Stathopoulos, N. A. \& Davazoglou, D. High performance organic light emitting diodes using substoichiometric tungsten oxide as efficient hole injection layer. Org. Electron. 13, 796-806 (2012).

120 Vasilopoulou, M., Palilis, L. C., Georgiadou, D. G., Douvas, A. M., Argitis, P., Kennou, S., Sygellou, L., Papadimitropoulos, G., Kostis, I., Stathopoulos, N. A. \& Davazoglou, D. Reduction of tungsten oxide: a path towards dual functionality utilization for efficient anode and cathode interfacial layers in organic light-emitting diodes. Adv Funct. Mater. 21, 1489-1497 (2011).

121 Vasilopoulou, M., Palilis, L. C., Georgiadou, D. G., Kennou, S., Kostis, I., Davazoglou, D. \& Argitis, P. Barrierless hole injection through sub-bandgap occupied states in organic light emitting diodes using substoichiometric moo $_{x}$ anode interfacial layer. Appl. Phys. Lett. 100, 013311 (2012).

122 Greiner, M. T., Helander, M. G., Wang, Z. B., Tang, W. M., Qiu, J. \& Lu, Z. H. A metallic molybdenum suboxide buffer layer for organic electronic devices. Appl. Phys. Lett. 96, 213302 (2010).

123 Chiam, S. Y., Dasgupta, B., Soler, D., Leung, M. Y., Liu, H., Ooi, Z. E., Wong, L. M. Jiang, C. Y., Chang, K. L. \& Zhang, J. Investigating the stability of defects in $\mathrm{moO}_{3}$ and its role in organic solar cells. Solar Energy Mater. Solar Cells 99, 197-203 (2012).

124 Vasilopoulou, M., Douvas, A. M., Georgiadou, D. G., Palilis, L. C., Kennou, S., Sygellou, L., Soultati, A., Kostis, I., Papadimitropoulos, G., Davazoglou, D. \& Argitis, P. The influence of hydrogenation and oxygen vacancies on molybdenum oxides work function and gap states for application in organic optoelectronics. J. Am. Chem. Soc. 134, 16178-16187 (2012).

125 Narioka, S., Ishii, H., Yoshimura, D., Sei, M., Ouchi, Y., Seki, K., Hasegawa, S. Miyazaki, T., Harima, Y. \& Yamashita, K. The electronic-structure and energy-leve alignment of porphyrin/metal interfaces studied by ultraviolet photoelectron-spectroscopy. Appl. Phys. Lett. 67, 1899-1901 (1995).

126 Egusa, S., Gemma, N., Miura, A., Mizushima, K. \& Azuma, M. Carrier injection characteristics of the metal organic junctions of organic thin-film devices. J. Appl. Phys. 71, 2042-2044 (1992).

127 Ishii, H. \& Seki, K. Energy level alignment at organic/metal interfaces studied by UV photoemission: breakdown of traditional assumption of a common vacuum level at the interface. IEEE Trans. Electron Devices 44, 1295-1301 (1997).

128 Hill, I. G., Rajagopal, A., Kahn, A. \& Hu, Y. Molecular level alignment at organic semiconductor-metal interfaces. Appl. Phys. Lett. 73, 662-664 (1998).

129 Harima, Y., Okazaki, H., Kunugi, Y., Yamashita, K., Ishii, H. \& Seki, K. Formation of Schottky barriers at interfaces between metals and molecular semiconductors of p- and n-type conductances. Appl. Phys. Lett. 69, 1059-1061 (1996). 
130 Heimel, G., Romaner, L., Bredas, J. L. \& Zojer, E. Interface energetics and level alignment at covalent metal-molecule junctions: pi-conjugated thiols on gold. Phys. Rev. Lett. 96, 196806 (2006).

131 Duhm, S., Gerlach, A., Salzmann, I., Broker, B., Johnson, R. L., Schreiber, F. \& Koch, N. PTCDA on $\mathrm{Au}(111), \mathrm{Ag}(111)$ and $\mathrm{Cu}(111)$ : correlation of interface charge transfer to bonding distance. Org. Electron. 9, 111-118 (2008).

$132 \mathrm{Koch}, \mathrm{N}$. Energy levels at interfaces between metals and conjugated organic molecules. J. Phys. Condes Matter 20, 184008 (2008).

133 Schlaf, R. Merritt, C. D., Picciolo, L. C. \& Kafafi, Z. H. Determination of the Orbital lineup at reactive organic semiconductor interfaces using photoemission spectroscopy. J. Appl. Phys. 90, 1903-1910 (2001).

134 Zou, Y., Kilian, L., Scholl, A., Schmidt, T., Fink, R. \& Umbach, E. Chemical bonding of ptcda on ag surfaces and the formation of interface states. Surf. Sci. 600 , 1240-1251 (2006).

135 Greczynski, G., Fahlman, M., Salaneck, W. R., Johansson, N., dos Santos, D. A. \& Bredas, J. L. Polymer interfaces studied by photoelectron spectroscopy: Li on polydioctylfluorene and $\mathrm{Alq}_{3}$. Thin Solid Films 363, 322-326 (2000).

136 Tautz, F. S. Structure and bonding of large aromatic molecules on noble metal surfaces: the example of PTCDA. Prog. Surf. Sci. 82, 479-520 (2007).

137 Demuth, J. E. \& Eastman, D. E. Photoemission observations of pi-d bonding and surface-reactions of adsorbed hydrocarbons on Ni(111). Phys. Rev. Lett. 32, $1123-1127$ (1974)

138 Kelemen, S. R. \& Fischer, T. E. Benzene and ethylene chemisorbed on transitionmetals - the measurement of energy-level shifts which accompany chemisorption. Surf. Sci. 102, 45-55 (1981).

139 Betti, M. G., Kanjilal, A. \& Mariani, C. Electronic states of a single layer of pentacene: standing-up and flat-lying configurations. J. Phys. Chem. A 111, 12454-12457 (2007).

140 Jung, M., Baston, U., Schnitzler, G., Kaiser, M., Papst, J., Porwol, T., Freund, H. J. \& Umbach, E. 21st European Congress on Molecular Spectroscopy (Eucmos 21): Molecular Spectroscopy and Molecular Structure 239-244 (Vienna, Austria, 1992).

141 Kleyna, R., Borgmann, D. \& Wedler, G. Adsorption and reaction of propene on $\mathrm{Ni}(100)$. Surf. Sci. 402, 131-134 (1998).

142 Sheu, B. R. \& Strongin, D. R. Adsorption and thermal-decomposition of methanol on 3d transition-metal aluminides - FeAl(110), NiAl and TiAl. J. Phys. Chem. 97, 10144-10151 (1993).

143 Ranke, W. \& Weiss, W. Adsorption and thermal decomposition of ethylbenzene and styrene on Pt(111) studied by UPS and XPS. Surf. Sci. 465, 317-330 (2000).

144 Duhm, S., Glowatzki, H., Cimpeanu, V., Klankermayer, J., Rabe, J. P., Johnson, R. L. \& Koch, N. Weak charge transfer between an acceptor molecule and metal surfaces enabling organic/metal energy level tuning. J. Phys. Chem. B. 110, 21069-21072 (2006).

145 Kahn, A., Koch, N. \& Gao, W. Y. Electronic structure and electrical properties of interfaces between metals and pi-conjugated molecular films. J. Polym. Sci. Pt B-Polym. Phys. 41, 2529-2548 (2003).

146 Ueno, N., Kera, S., Sakamoto, K. \& Okudaira, K. K. Energy Band and electronvibration coupling in organic thin films: photoelectron spectroscopy as a powerful tool for studying the charge transport. Appl. Phys. A Mater. Sci. Processing 92, 495-504 (2008).

147 Koch, N., Heimel, G., Wu, J. S., Zojer, E., Johnson, R. L., Bredas, J. L., Mullen, K. \& Rabe, J. P. Influence of molecular conformation on organic/metal interface energetics. Chem. Phys. Lett. 413, 390-395 (2005).

148 Fukagawa, H., Yamane, H., Kataoka, T., Kera, S., Nakamura, M., Kudo, K. \& Ueno, N. Origin of the highest occupied band position in pentacene films from ultraviolet photoelectron spectroscopy: hole stabilization versus band dispersion. Phys. Rev. B. 73, 245310 (2006).

149 Kera, S., Setoyama, H., Onoue, M., Okudaira, K. K., Harada, Y. \& Ueno, N. Origin of indium-perylene-3,4,9,10-tetracarboxilic dianhydride interface states studied by outermost surface spectroscopy using metastable atoms. Phys. Rev. B. 63, 115204 (2001).

150 Kera, S., Fukagawa, H., Kataoka, T., Hosoumi, S., Yamane, H. \& Ueno, N. Spectroscopic evidence of strong pi-pi interorbital interaction in a lead-phthalocyanine bilayer film attributed to the dimer nanostructure. Phys. Rev. B. 75, 121305 (2007).

151 Kuppers, J., Michel, H., Nitschke, F., Wandelt, K. \& Ertl, G. Xenon adsorption as a tool for local surface-structure determination at $\operatorname{Ir}(100)$ surfaces. Surf. Sci. 89, 361-369 (1979).

152 Jacobi, K. \& Rotermund, H. H. UV photoemission from physisorbed atoms and molecules - electronic binding-energies of valence levels in monolayers and multilayers. Surf. Sci. 116, 435-455 (1982).

153 Jacobi, K., Hsu, Y. P. \& Rotermund, H. H. Photoemission from Ne, Ar, Kr and Xe layers on $\mathrm{Ni}(110)$ and Ga films. Surf. Sci. 114, 683-691 (1982).

154 Rotermund, H. H. \& Jacobi, K. Physisorption on a low work function metal - ARUPS from xenon on cesium. Surf. Sci. 126, 32-40 (1983).

155 Kuppers, J., Nitschke, F., Wandelt, K. \& Ertl, G. Adsorption of Xe on Pd(110). Surf. Sci. 87, 295-314 (1979)

156 Kuppers, J., Wandelt, K. \& Ertl, G. Influence of the local surface-structure on the $5 p$ photoemission of adsorbed xenon. Phys. Rev. Lett. 43, 928-931 (1979).

157 Wandelt, K. \& Hulse, J. E. Xenon adsorption on palladium.1. The homogeneous (110), (100), and (111) surfaces. J. Chem. Phys. 80, 1340-1352 (1984).

$158 \mathrm{Hu}$, P., King, D. A., Lee, M. H. \& Payne, M. C. Orbital mixing in co chemisorption on transition-metal surfaces. Chem. Phys. Lett. 246, 73-78 (1995).
159 Hammer, B., Morikawa, Y. \& Norskov, J. K. Co chemisorption at metal surfaces and overlayers. Phys. Rev. Lett. 76, 2141-2144 (1996).

160 Jones, P. M., May, J. A. \& Solomon, E. I. Carbonyl bonding on $\mathrm{ZnO}(0001), \mathrm{CuCl}(111)$ and $\mathrm{Cu}_{2} \mathrm{O}(111) 3 \mathrm{~d}(10)$ metal ion surfaces: photoelectron spectroscopy of $\mathrm{co} / \mathrm{Cu}_{2} \mathrm{O}(111)$ and electronic structure calculations. Inorganica. Chim. Acta. 276, 327-333 (1998).

161 Wong, Y. T. \& Hoffmann, R. Chemisorption of Carbon-monoxide on 3 metal-surfaces $\mathrm{Ni}(111), \mathrm{Pd}(111)$, and Pt(111) - a comparative-study. J. Phys. Chem. 95, 859-867 (1991).

162 Rhodin, T. N., Kanski, J. \& Brucker, C. Photoemission study of coverage-dependent chemisorption interaction between Co and $\operatorname{Ir}(111)$. Solid State Commun. 23, 723-727 (1977).

163 Bech, L., Onsgaard, J., Hoffmann, S. V. \& Godowski, P. J. Co dissociation on K-modified $\mathrm{Cu}(112)$ and $\mathrm{Cu}(117)$. Surf. Sci. 482, 243-249 (2001).

164 Freund, H. J. \& Neumann, M. Photoemission of molecular adsorbates. Appl. Phys. A Mater. Sci. Processing. 47, 3-23 (1988).

165 Kanski, J. \& Rhodin, T. N. Chemisorptive bonding and reaction of nitric-oxide on $\operatorname{Ir}(100)$ and $\operatorname{Ir}(111)$ surfaces. Surf. Sci. 65, 63-76 (1977).

166 Henderson, M. A. The interaction of water with solid surfaces: fundamental aspects revisited. Surf. Sci. Rep. 46, 1-308 (2002).

167 Weiss, W. \& Ranke, W. Surface chemistry and catalysis on well-defined epitaxial ironoxide layers. Prog. Surf. Sci. 70, 1-151 (2002).

168 Vazquez, H., Flores, F., Oszwaldowski, R., Ortega, J., Perez, R. \& Kahn, A. Barrier formation at metal-organic interfaces: dipole formation and the charge neutrality level. Appl. Surf. Sci. 234, 107-112 (2004).

169 Vazquez, H., Gao, W., Flores, F. \& Kahn, A. Energy level alignment at organic heterojunctions: role of the charge neutrality level. Phys Rev B. 71, 041306 (2005).

170 Vazquez, H., Flores, F. \& Kahn, A. Induced density of states model for weaklyinteracting organic semiconductor interfaces. Org. Electron. 8, 241-248 (2007).

171 Vazquez, H., Dappe, Y. J., Ortega, J. \& Flores, F. A unified model for metal/organic interfaces: idis, 'pillow' effect and molecular permanent dipoles. Appl. Surf. Sci. 254, 378-382 (2007).

172 Koch, N. \& Vollmer, A. Electrode-molecular semiconductor contacts: work-functiondependent hole injection barriers versus fermi-level pinning. Appl. Phys. Lett. 89, 162107 (2006).

173 Tengstedt, C., Osikowicz, W., Salaneck, W. R., Parker, I. D., Hsu, C. H. \& Fahlman, M. Fermi-level pinning at conjugated polymer interfaces. Appl. Phys. Lett. 88, 053502 (2006).

174 Greiner, M. T., Helander, M. G., Tang, W. M., Wang, Z. B., Qiu, J. \& Lu, Z. H. Universal energy-level alignment of molecules on metal oxides. Nat. Mater. 11, 76-81 (2012).

175 Ley, L., Smets, Y., Pakes, C. I. \& Ristein, J. Calculating the universal energy-level alignment of organic molecules on metal oxides. Adv. Funct. Mater. 23, 794-805 (2013).

176 Crispin, A., Crispin, X., Fahlman, M., Berggren, M. \& Salaneck, W. R. Transition between energy level alignment regimes at a low band gap polymer-electrode interfaces. Appl. Phys. Lett. 89, 213503 (2006).

177 Osikowicz, W., de Jong, M. P. \& Salaneck, W. R. Formation of the interfacial dipole at organic-organic interfaces: $\mathrm{C}_{60}$ /polymer interfaces. Adv. Mater. 19, 4213-4217 (2007).

178 Fukagawa, H., Kera, S., Kataoka, T., Hosoumi, S., Watanabe, Y., Kudo, K. \& Ueno, N. The role of the ionization potential in vacuum-level alignment at organic semiconductor interfaces. Adv. Mater. 19, 665-668 (2007).

179 Lange, I., Blakesley, J. C., Frisch, J., Vollmer, A., Koch, N. \& Neher, D. Band bending in conjugated polymer layers. Phys. Rev. Lett. 106, 216402 (2011).

180 Henrich, V. E. \& Cox, P. A. The Surface Science of Metal Oxides (Cambridge University Press, Cambridge, 1994).

181 Asahi, R., Taga, Y., Mannstadt, W. \& Freeman, A. J. Electronic and optical properties of anatase $\mathrm{TiO}_{2}$. Phys. Rev. B. 61, 7459-7465 (2000).

182 Jiang, B., Zuo, J. M., Jiang, N., O'Keeffe, M. \& Spence, J. C. H. Charge density and chemical bonding in rutile, $\mathrm{TiO}_{2}$. Acta. Crystallogr. A59, 341-350 (2003).

183 Mott, N. F. The transition to the metallic state. Philos. Mag. 6, 287-309 (1961).

184 Hubbard, J. Electron correlations in narrow energy bands. Proc. R. Soc. Lond. A 276 238-257 (1963)

185 Hubbard, J. Electron correlations in narrow energy bands II. The degenerate band case. Proc. R. Soc. Lond. A 277, 237-259 (1964).

186 Hubbard, J. Electron correlations in narrow energy bands III3. An improved solution. Proc. R. Soc. Lond. A 281, 401-419 (1964).

187 Imada, M., Fujimori, A. \& Tokura, Y. Metal-insulator transitions. Rev. Mod. Phys. 70, 1039-1263 (1998).

188 Zaanen, J., Sawatzky, G. A. \& Allen, J. W. Band-gaps and electronic-structure of transition-metal compounds. Phys. Rev. Lett. 55, 418-421 (1985).

189 Scanlon, D. O., Morgan, B. J., Watson, G. W. \& Walsh, A. Acceptor levels in p-type $\mathrm{Cu}_{2} \mathrm{O}$ : rationalizing theory and experiment. Phys. Rev. Lett. 103, 096405 (2009).

190 Nolan, M. \& Elliott, S. D. The p-type conduction mechanism in $\mathrm{cu}_{2} \mathrm{O}$ : a first principles study. Phys. Chem. Chem. Phys. 8, 5350-5358 (2006).

191 Lany, S., Osorio-Guillen, J. \& Zunger, A. Origins of the doping asymmetry in oxides: hole doping in NiO versus electron doping in ZnO. Phys. Rev. B 75, 241203 (2007).

192 Raebiger, H., Lany, S. \& Zunger, A. Origins of the p-type nature and cation deficiency in $\mathrm{Cu}_{2} \mathrm{O}$ and related materials. Phys. Rev. B 76, 045209 (2007).

193 Fergus, J. W. Doping and defect association in oxides for use in oxygen sensors. J. Mater. Sci. 38, 4259-4270 (2003).

194 Matsui, T. \& Naito, K. Electrical-conductivity, oxygen potentials and defect structures of pure $\mathrm{UO}_{2+\mathrm{x}}$ and $\mathrm{UO}_{2+\mathrm{x}}$ doped with various cations. J. Less-Common Metals 121, 279-283 (1986). 
195 Nerikar, P., Watanabe, T., Tulenko, J. S., Phillpot, S. R. \& Sinnott, S. B. Energetics of intrinsic point defects in uranium dioxide from electronic-structure calculations. J. Nucl. Mater. 384, 61-69 (2009).

196 Ratcliff, E. L., Zacher, B. \& Armstrong, N. R. Selective inter layers and contacts in organic photovoltaic cells. J. Phys. Chem. Lett. 2, 1337-1350 (2011).

197 Tao, C., Ruan, S., Xie, G., Kong, X., Shen, L., Meng, F., Liu, C., Zhang, X., Dong, W. \& Chen, W. Role of tungsten oxide in inverted polymer solar cells. Appl. Phys. Lett. 94, 043311 (2009).

198 Steim, R., Choulis, S. A., Schilinsky, P. \& Brabec, C. J. Interface modification for highly efficient organic photovoltaics. Appl. Phys. Lett. 92, 093303 (2008).

199 Stubhan, T., Ameri, T., Salinas, M., Krantz, J., Machui, F., Halik, M. \& Brabec, C. J. High shunt resistance in polymer solar cells comprising a $\mathrm{MoO}_{3}$ hole extraction layer processed from nanoparticle suspension. Appl. Phys. Lett. 98, 253308 (2011).

200 Steirer, K. X., Ndione, P. F., Widjonarko, N. E., Lloyd, M. T., Meyer, J., Ratcliff, E. L., Kahn, A., Armstrong, N. R., Curtis, C. J., Ginley, D. S., Berry, J. J. \& Olson, D. C. Enhanced efficiency in plastic solar cells via energy matched solution processed $\mathrm{NiO}_{\mathrm{x}}$ interlayers. Adv. Energy Mater. 1, 813-820 (2011).

201 Waldauf, C., Morana, M., Denk, P., Schilinsky, P., Coakley, K., Choulis, S. A. \& Brabec, C. J. Highly efficient inverted organic photovoltaics using solution based titanium oxide as electron selective contact. Appl. Phys. Lett. 89, 233517 (2006).

202 Wong, K. H., Ananthanarayanan, K., Luther, J. \& Balaya, P. Origin of hole selectivity and the role of defects in low-temperature solution-processed molybdenum oxide interfacial layer for organic solar cells. J. Phys. Chem. C 116, 16346-16351 (2012).

203 Kyaw, A. K. K., Sun, X. W., Jiang, C. Y., Lo, G. Q., Zhao, D. W. \& Kwong, D. L. An inverted organic solar cell employing a sol-gel derived $\mathrm{ZnO}$ electron selective layer and thermal evaporated $\mathrm{moO}_{3}$ hole selective layer. Appl. Phys. Lett. 93, 221107 (2008).

204 Smoluchowski, R. Anisotropy of the electronic work function of metals. Phys. Rev. 60, 661-674 (1941).

$205 \mathrm{Li}$, W. \& Li, D. Y. On the correlation between surface roughness and work function in copper. J. Chem. Phys. 122, 064708 (2005).

206 Strayer, R. W., Mackie, W. \& Swanson, L. W. Work function measurements by fieldemission retarding potential method. Surf. Sci. 34, 225-248 (1973).

207 Lin, Y. -J., Baikie, I. D., Chou, W. -Y., Lin, S. -T., Chang, H. -C., Chen, Y. -M. \& Liu, W. $-F$. Changes in surface roughness and work function of indium-tin-oxide due to $\mathrm{KrF}$ excimer laser irradiation. J. Vacuum Sci. Technol. A 23, 1305-1308 (2005).

208 Gutmann, S., Wolak, M. A., Conrad, M., Beerbom, M. M. \& Schlaf, R. Effect of ultraviolet and $\mathrm{X}$-ray radiation on the work function of $\mathrm{TiO}_{2}$ surfaces. J. Appl. Phys. 107, 103705 (2010)

209 Jablonski, A. \& Wandelt, K. Quantitative aspects of ultraviolet photoemission of adsorbed xenon - a review. Surf. Interface Anal. 17, 611-627 (1991).

$210 \mathrm{Li}, \mathrm{K}$. Y. \& Xue, D. F. Estimation of electronegativity values of elements in different valence states. J. Phys. Chem. A 110, 11332-11337 (2006).
211 Mulliken, R. S. A new electroaffinity scale; together with data on valence states and on valence ionization potentials and electron affinities. J. Chem. Phys. 2, 782-793 (1934).

212 Matar, S. F., Campet, G. \& Subramanian, M. A. Electronic properties of oxides: chemical and theoretical approaches. Prog. Solid State Chem. 39, 70-95 (2011).

213 Nethercott, A. Prediction of fermi energies and photoelectric thresholds based on electronegativity concepts. Phys. Rev. Lett. 33, 1088-1091 (1974).

214 Campet, G., Portier, J. \& Subramanian, M. A. Electronegativity versus fermi energy in oxides: the role of formal oxidation state. Mater. Lett. 58, 437-438 (2004).

215 Neamen, D. Semiconductor Physics and Devices. 3rd edn Ch. 4. (McGraw-Hill, New York, 2003).

216 Greiner, M. T., Helander, M. G., Wang, Z. -B., Tang, W. -M. \& Lu, Z. -H. Effects of processing conditions on the work function and energy-level alignment of nio thin films. J. Phys. Chem. C 114, 19777-19781 (2010).

217 Noh, S., Suman, C. K., Lee, D., Kim, S. \& Lee, C. Study of buffer layer thickness on bulk heterojunction solar cell. J. Nanosci. Nanotechnol. 10, 6815-6818 (2010).

$218 \mathrm{Wu}$, J., Guo, X. -Y. \& Xie, Z. -Y. Effects of the molybdenum oxide/metal anode interfaces on inverted polymer solar cells. Chin. Phys. Lett. 29, 098801 (2012).

219 Wang, Y. -Z., Yang, M., Qi, D. -C., Chen, S., Chen, W., Wee, A. T. S., Gao, X. -Y. \& Synchrotron-Based, A. Photoemission study of the $\mathrm{MoO}_{3} / \mathrm{Co}$ interface. J. Chem. Phys. 134, 034706 (2011).

220 Kanai, K., Koizumi, K., Ouchi, S., Tsukamoto, Y., Sakanoue, K., Ouchi, Y. \& Seki, K. Electronic structure of anode interface with molybdenum oxide buffer layer. Org. Electron. 11, 188-194 (2010).

221 Helander, M. G., Wang, Z. B., Greiner, M. T., Qiu, J. \& Lu, Z. H. Substrate dependent charge injection at the $\mathrm{v}_{2} \mathrm{O}_{5}$ /organic interface. Appl. Phys. Lett. 95, 083301 (2009).

222 Ifran, I., Ding, H., Gao, Y., Kim, D. Y., Subbiah, J. \& So, F Energy level evolution of molybdenum trioxide interlayer between indium tin oxide and organic semiconductor. Appl. Phys. Lett. 96, 073304 (2010).

223 Wang, Y. Z., Cao, L., Qi, D. C., Chen, W., Wee, A. T. S. \& Gao, X. Y. Tuning the interfacial hole injection barrier between p-type organic materials and Co using a $\mathrm{MoO}_{3}$ buffer layer. J. Appl. Phys. 112, 033704 (2012).

224 Greiner, M. T., Chai, L., Helander, M. G., Tang, W.-M. \& Lu, Z. -H. Metal/metal-oxide interfaces: how metal contacts affect the work function and band structure of $\mathrm{MoO}_{3}$. Adv. Funct. Mater. 23, 215-226 (2013).

(c) (1) (2) This work is licensed under a Creative Commons Attribution-NonCommercial-ShareAlike 3.0 Unported License. To view a copy of this license, visit http://creativecommons. org/licenses/by-nc-sa/3.0/

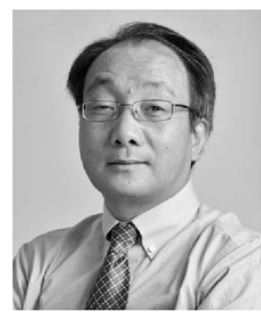

Dr Zheng-Hong Lu is a full professor and a Tier I Canada Research Chair in Organic Optoelectronics at the University of Toronto. He received a $\mathrm{PhD}$ degree in engineering physics in 1990 from the University of Montreal, Canada. Prior to his current appointment, he was employed by the National Research Council (NRC) as an assistant and then an associate research officer. While at NRC he developed a number of materials and processes for microelectronics and optoelectronics, in particular light-emitting silicon superlattices, dielectrics for silicon transistors, and surface passivation for solid-state lasers. In 1998, he moved to the University of Toronto to create an Organic Optoelectronics Research Group. His group's research includes OLED materials and device engineering for flat-panel display and solid-state lighting applications. He has authored and co-authored over 250 publications in technical journals and has filed more than 20 patent applications.

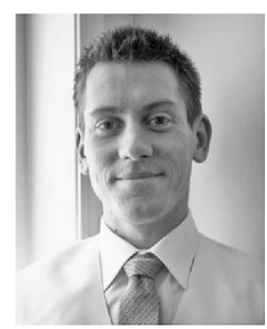

Dr Mark Greiner, received a Bachelors of Science and Masters of Science in Chemistry from McMaster University in 2008. He received a $\mathrm{PhD}$ degree in the Department of Materials Science and Engineering at the University of Toronto in 2012, under the supervision of Prof. Zheng-Hong Lu. Mark's thesis work was focused on the electronic properties of transition metal oxides and how they affect energy-level alignment with organic semiconductors. In May 2012, Mark joined the Department of Inorganic Chemistry at the Fritz-Haber-Institute of the Max-Planck Society to conduct post-doctoral research on metal oxides in catalysis. 